\title{
Dispositif simple \\ pour réduire la célérité des ondes élastiques dans les conduites en charge*
}

\section{Application à la protection contre certains coups de bélier}

\author{
Simple device for reducing the celerity of elasticity \\ waves in pipes; \\ application to protection against certain \\ waterhammer effects
}

\author{
PAR G. REMENTERAS

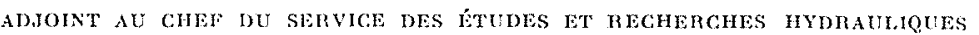 \\ D'ÉLECTURCTT'́ DE FRANCE
}

English synopsis, p. 115.

\section{INTRODUCTION}

L'objet de la présente note est de rechercher les possibilités pratiques de réduire la valeur maximum du coup de bélier dans une conduite en augmentant l'élasticité de l'ensemble «conduite-liquide transporté », notamment par insertion à l'intérieur et sur toute la longueur de celle-ci, d'un élément élastique très déformable tel qu'un tuyau à parois très souples rempli d'air comprimé.

Dans le chapitré I nous étudions, suivant la théorie d'Allievi, l'influence d'une diminution de la célérité sur les phénomènes de coups de

$\left(^{*}\right)$ Ce dispositif fait l'objet du brevet français $\mathrm{n}^{\circ}$ P.V, 616.279 délivié le 14-9-1951 à Electricité de France, bélier dans une conduite à caractéristique unique.

Dans le chapitre II, après avoir décrit sommairement quelques dispositifs réducteurs de célérité, nous établissons les formules permettant le calcul de la célérité dans une conduite comportant un tuyau anti-bélier schématique à compression d'air.

Le chapitre III est un bref compte rendu des essais effectués au Laboratoire de Chatou sur un troncon de pipe-line de $240 \mathrm{~mm}$ de diamètre et $90 \mathrm{~m}$ de longueur pour vérifier ces formules.

En conclusion, nous donnons quelques indications sur le champ d'application et les limites d'emploi pratiques des dispositifs réducteurs de célérité envisagés plus haut. 


\section{CHAPITRE I}

\section{INFLUENCE D'UNE DIMINUTION DE LA CELERITE SUR LES PHENOMENES DE COUP DE BELIER DANS UNE CONDUITE A CARACTERISTIQUE UNIQUE}

\section{I. - Variation de pression à l'obturateur} (vanne, distributeur, etc.) dans le cas d'une fermeture linéaire complète de ce dernier.

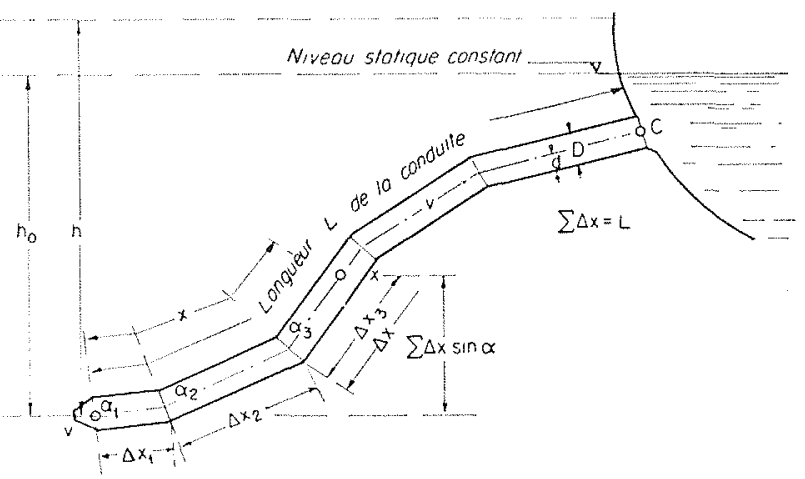

Fig. 1.

Soient (fig. 1) :

$x$ l'abscisse, mesurée à partir de l'extrémité aval, d'une section de la conduite de longueur totale $\mathrm{L}$;

$h_{i x}$ la pression, exprimée en hauteur d'eau, à l'abscisse $x$ et au temps $i$, compté à partir du début de la perturbation créant le coup de bélier;

$h_{0 . w}$ la pression, en ce même point, à l'instant 0 , c'est-à-dire en " régime permanent $\gg$;

$h_{i x}-h_{0 x}$ la surpression ou coup de bélier en $x$ à l'instant $i$

$V_{i, x}$ et $V_{0, x}$ les vitesses dans la section d'abscisse $x$ respectivement à l'instant $i$ et $\grave{a}$ l'instant initial;

a la célérité des ondes élastiques parcourant la conduite, telle qu'elle résulte de la formule :

$$
\frac{1}{a^{2}}=\frac{\sigma}{g}\left(\frac{1}{\varepsilon}+\frac{\mathrm{D}}{\mathrm{E} e}\right)
$$

D est le diamètre de la conduite;

$e$ son épaisseur;
E le module d'élasticité du matériau qui la constitue;

$\frac{1}{\varepsilon}$ le coefficient de compressibilité de $y==\frac{2 L}{a}$ la durée de phase de la conduite.

Nous utiliserons souvent des « grandeurs relatives », c'est-à-dire sans dimensions, telles que :

$$
\begin{gathered}
y=\frac{h_{\mathrm{i}}}{h_{0}}: \text { pression relative } \\
y-1=\frac{h_{i}-h_{0}}{h_{0}}: \text { surpression relative }
\end{gathered}
$$

et surtout la caractéristique d'Alliévi de la conduite :

$$
\rho=\frac{a V_{0}}{2 g h_{0}}
$$

$V_{0}$ et $h_{0}$ sont les valeurs de la vitesse et de la pression à l'obturateur à l'instant 0 .

La caractéristique d'Alliḱvr associée au temps relatif de fermeture : $\theta=\frac{\dddot{\theta}}{\mu}$ permet de calculer la loi de variation de la pression relative $y=f(t)$ au droit de l'obturateur - et par récurrence tout le long de la conduite - au cours du coup de bélier.

Le phénomène prend deux aspects nettement

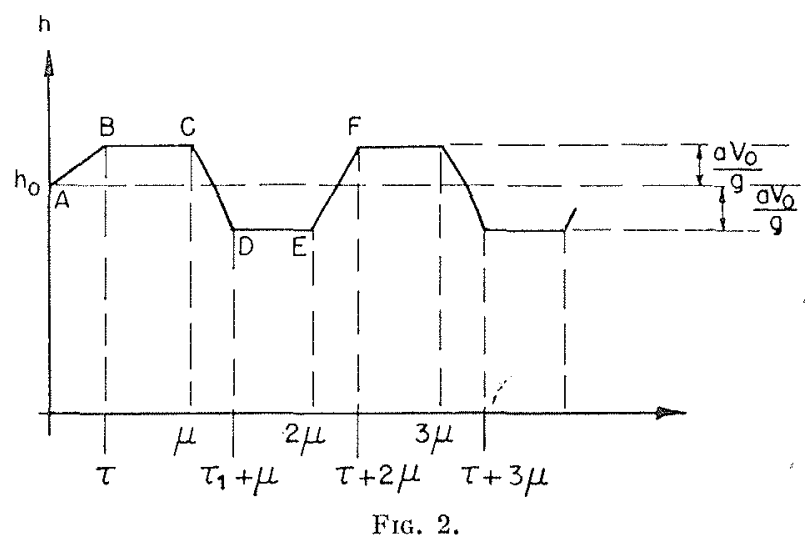


différents, suivant que le temps de fiermeture $\widetilde{6}$ de l'obturateur est inférieur ou supérieur à la durée de phase $\mu=\frac{2 L}{a}$ de la condiite.

a) Fermetures rapides :

$$
\widetilde{\sigma}<\frac{2 \mathrm{~L}}{a} \text { c'est-à-dire } \theta<1
$$

La courbe $y=f(t)$ présente l'allure indiquée par la figure 2.

La surpression atteint au temps $\approx$ son maximum égal à :

$$
h-h_{0}=\frac{a \mathrm{~V}_{0}}{g}
$$

soit en «grandeurs relatives»:

$$
y=1+2 \text { ? }
$$

Ce maximum est donc indépendant du temps de fermeture :

$$
\because<\frac{2 \mathrm{~K}}{a}
$$

Supposons que, par un dispositif convenable, nous réduisions la célérité $a$ à une valeur $a^{\prime}=\frac{a}{\alpha}$. Le maximum du coup de bélier sera évidemment divisé par a et ramené à :

$$
h-h_{0}=\frac{a^{\prime} \mathrm{V}_{0}}{g}=\frac{1}{\alpha} \frac{a \mathrm{~V}_{n}}{g}
$$

soit :

$$
y-1=2 q^{\prime}=\frac{2 \rho}{\alpha}
$$

\section{b) Femmetures lentes :}

$$
\widetilde{\theta}>\frac{2 \mathrm{~L}}{a} \text { c'est-à-dire } 0>1
$$

La méthode d'Altı́́vi permet de calculer anaIytiquement tout d'abord les pressions relatives $y_{1}, y_{2} \ldots y_{i}$ aux instants., $2 \ldots . . i y_{1}$, dits « instants de rythmes entiers $\gg$ et par divers artifices aux « instants de rythmes intercalaires ». On peut donc tracer par points la courbe $y=f(t)$.

En outre, Alurívi a montré que les valeurs des surpressions $y_{1}, y_{2}, y_{1}$ tendent toujour's vers une limile $y_{m}$, que ce soit par valeurs croissantes ou par valeurs alternativement plus grandes ou plus petites que $y_{m}$; cette limite est donnée par l'équation :

$$
\sqrt{y_{m}}=\frac{p}{20}+\sqrt{\left(\frac{p}{20}\right)^{2}+1}
$$

Des études systématiques remarquables du même auteur ont permis de classer les courbes de pression $y=f(t)$ en un certain nombre de types dont les figures 3,4 et 5 représentent les plus importants en pratique.

On voit que le maximum de la pression pent se produire soit à la fin de la première phase

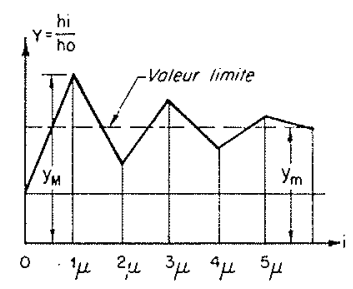

Fig. 3.

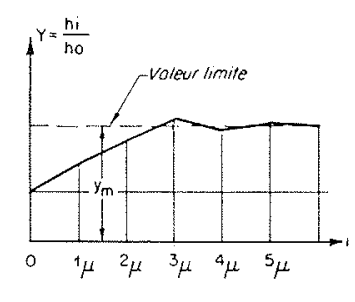

Fig. 4.

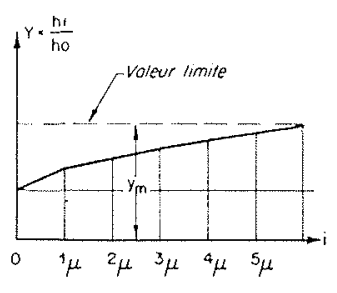

FxG. 5. (fig. 3), soit pendant ou à la fin de l'une des phases suivantes (fig. 4 et 5 ).

Comme tout le phénomène dépend des grandeurs relatives et $\theta$, on peut construire des abaques ( synopsis d'Alrmey », abaques de M. GARIEL, etc...) qui donnent, en fonction de ? et 0 , non seulement la valeur du maximum $y_{m}$, mais la phase au cours ou à la fin de laquelle se produit ce maximum et aussi la « pression limite» $y_{m}$.

Pour le problème qui nous occupe, l'abaque de M. Schlat paraît particulièrement bien adapté; nous en rappelons ci-après le principe. c) Abaque de M. Scmlag:

M. Schlag établit un diagramme cartésien (fig. 6) en prenant comme abscisse $\frac{\rho}{\theta}$ (au lieu de $\%$ dans le synopsis d'Alıŕvir).

Il trace d'abord, en fonction de $\frac{\rho}{0}$, la courbe de la pression relative limite :

$$
\sqrt{y_{m}}=\frac{?}{20}+\sqrt{\left(\frac{0}{20}\right)^{2}+1}
$$

diagramme qui illustre la propriété de la pres- 


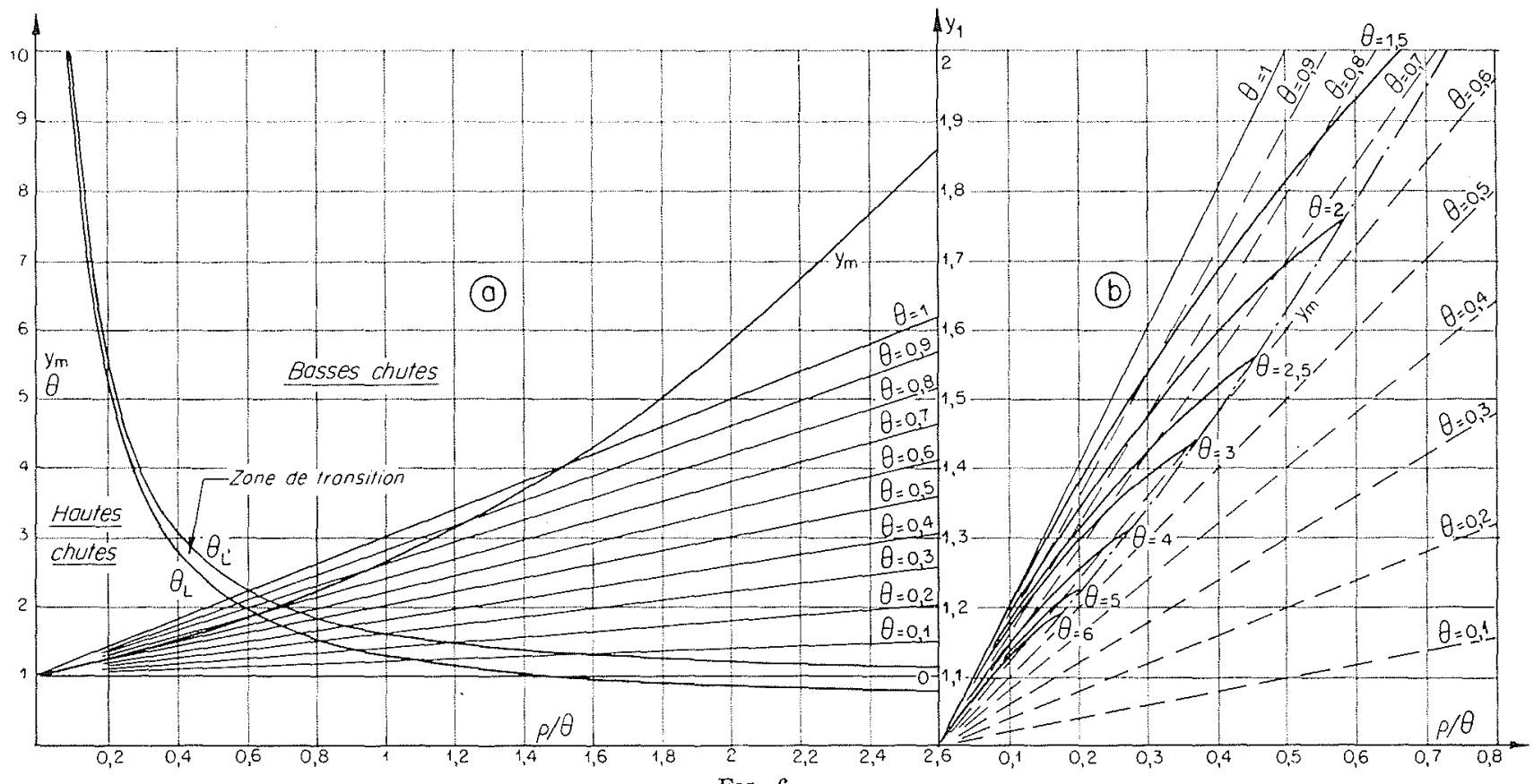

FIG. 6.

sion limite relative d'ètre fonction uniquement du rapport $\frac{\text { ? }}{\theta}$.

La pression limite $y_{m}$ étant ainsi déterminée, il convient de compléter l'abaque par des courbes donnant la valeur de la pression relative maximum $y_{\mathrm{M}}$.

\section{1. - Fermetures rapides :}

$$
0<1
$$

Nous avons vu que la pression relative maximum s'exprimait par :

$$
y=1+2 ?=1+\frac{2 ?}{\theta} 0
$$

Pour chaque valeur $\theta_{1}$, la fonction $y=f\left(\frac{0}{0},{ }^{\circ}\right)$ est donc une droite cotée $\theta_{i}$ passant par le point $(0,1)$.

\section{2. - Fermetures lentes :}

Le problème est ici plus compliqué puisque la valeur du maximun de pression dépend de la courbe-type des figures 3,4 et 5 a laquelle se rattache le coup de bélier étudié.

M. Schlag montre que si :

$$
0<\theta_{\mathrm{L}}=\frac{1}{2} \frac{2 \rho-1}{2 \rho-2}
$$

on se trouve dans la zone du diagramme qu'il appelle «zone des hautes chutes » qui correspond à une courbe $y=f(t)$ du type représenté dans la figure 3.

Si :

$$
0>{ }^{i} L_{1}=\frac{p^{2}}{p^{2-1}}
$$

on se trouve dans la zone des "basses chutes》 qui correspond à une courbe $y=f(t)$ du type représenté dans la figure 5.

Enfin, pour les valeurs de o comprises dans la zone - fort étroite en pratique - dite région de transition comprise entre la région des hautes chutes et celles des basses chutes, $y=f(t)$ présente l'allure de la courbe de la figure 4.

On trace done sur le diagramme, à partir des équations ci-dessus, les deux courbes auxiliaires $\theta_{\mathrm{L}}$ et $\theta_{\mathrm{L}}$ qui donnent, en fonction de $\frac{?}{\theta}$, les temps de fermeture $\theta_{\mathrm{L}}$ et $\theta_{\mathrm{L}}$, qui limitent les régions ci-dessus.

Dans la « région des hautes chutes », $y_{31}$ est égal (ou exceptionnellement très voisin) à la pression $y_{1}$ à la fin de la première phase (c'est-à̀dire à l'instant $i=\mu$ et l'on a, pour les coups de bélier pas trop importants $\left(y_{\mathrm{M}} \leqslant 1,50\right)$ :

$$
y_{1}=y_{\mathrm{M}}=\frac{2 \rho}{\theta} \frac{1}{1+\frac{\rho}{\theta}(0-1)}
$$


Le diagramme $b$ (fig. 6) donne, à grande échelle, le faisceau des courbes (cotées en 0 ) correspondant à la fonction $y=f\left(\frac{p}{\theta}, 0\right)$ ci-dessus; on voit que chacune des courbes $y_{1}\left(\frac{\theta}{\theta}\right)$ s'ariete à une valeur de $\frac{\rho}{\theta}$ bien détermince; c'est qu'audela de cette valeur (pour la valeur particuliere de 0 afférente à la courbe), cesse la région des hautes chutes; on peut d'ailleurs voir que cetle valeur extrême de $y_{1}$ est égale à $y_{m}$ (courbe en trail mixte).

Dans la « région des basses chutes 》 on admettra, en pratique, que le coup de bélier maximum $y_{\mathrm{M}}$ est égal au coup de bélier limite $y_{m}$, ce qui donne, pour la surpression $y_{m}-1$, lorsque le coup de bélier reste dans les limites de la pratique industrielle (de SPARRE) :

$$
y_{m}-1=\frac{\rho}{\theta} \cdot \frac{2}{2-\frac{\theta}{\theta}}
$$

d) INFLUENGE D'UNE RÉDUCTION DE LA CÉLÉRITÉ $a$ :

Supposons que, pax un dispositif tel que ceux décrits au chapitre II ci-apres, nous réduisions la célérité $a$ de la conduite à la valeur $a^{\prime}=\frac{a}{\alpha}$. Toutes choses (et en particulier le temps de fermeture $\mathscr{O}$ ) restant égales d'ailleurs :

- La durce de phase :

$$
\mu=\frac{2 \mathrm{~L}}{a} \text { deviendra }: \mu^{\prime}=x p
$$

- Le temps relatif de fermeture:

$$
\theta=\frac{\widetilde{G}}{\mu_{*}} \text { deviendra }: \theta^{\prime}=\frac{\theta}{\alpha}
$$

- La caractéristique d'Altóver :

$$
?=\frac{a V_{0}}{2 g h_{0}} \text { deviendra }: o^{\prime}=\frac{?}{\alpha}
$$

- Le paramètre $\frac{e}{\theta}$, de M. Schlar, sera incliangé :

$$
\frac{\rho}{0}=\frac{\rho^{\prime}}{\theta^{\prime}}
$$

La modification corrélative des coups de bélier «limites » et « maxima » s'en déduit facilement à la lumière des formules données plus haut et de l'abaque de M. Schlag; on arrive aux résultats que nous résumons ci-après :

Dans le cas des fermetures rapides, la sur- pression maximum est réduite dans le rapport $\alpha=\frac{a}{a^{\prime}}$ des célérités:

$$
y-1=2 q^{\prime}=\frac{2 p}{\alpha}
$$

Dans le cas des fermetures lentes, la surpression maximum est angmentée dans la « région des hautes chutes (abaque $b$ de la fig. 6) et demeure inchangée dans la « région des basses chutes $\gg$.

Mais il peut se produire que la réduction de $a$ a $a^{\prime}$ de la célérité conduise, pour une mème duré absolue de fermeture $\ddot{\circ}$ à transformer une fermeture lente en une fermeture rapide; il suflit pour cela que a soit supérieur à 0 , ce qui entraine $\theta^{\prime}=\frac{\theta}{\alpha}<1$.

Un simple coup d'cil sur l'abaque de M. Schlag permet de se rendre compte de l'inlérêt de la réduction de célérité pour une conduite caractérisée par le paramètre p et pour une durée relative de fermeture 0 . Les valeurs de $\frac{\theta}{\theta}$ lues en abscisse sont les mêmes pour les deux conduites; les coups de bélier maxima, de « fermeture rapide », sont donnés par les faisceaux de droites cotés en $\theta^{\prime}$; la réduction de célérité est avantageuse dans toute la zone où ces faisceaux se trouvent au-dessous de la courbe $y_{\text {in }}$ (abaque $[a]$ ) ou du faisceau de courbes cotées en $\theta$ (abaque $[b]$ ).

Des calculs simples montrent que la réduction de célérité entraine une diminution de la surpression maximum si on a :

$$
\begin{gathered}
\alpha>0+0(0-1) \text { dans la région des } \ll \text { hautes } \\
\text { chutes } »
\end{gathered}
$$

et :

$$
\begin{gathered}
x>20-? \quad \text { dans la région des «basses } \\
\text { chutes } .
\end{gathered}
$$

II. - Valeur maximum possible du coup
de bélier dans une installation donnée,
compte tenu des "mancuvres partielles"

\section{a) Dragramme de M. Gariel.}

Les formules et abaques donnés ci-dessus permettent de calculer le coup de bélier maximum au droit de l'obturateur pour toutes les conduites

1. C'est cette valeur qui conditionne le dimensionnement de la conduite, au moins au droit de l'obturateur. 
et toutes les manouvres linéaires caractérisées par les paramètres e et 0 .

En particulier, ils donnent le moyen de rechercher « la manœuvre la plus dangereuse » pouvant être réalisée dans une installation donnée.

Dans l'hypothèse classique ${ }^{1}$ d'une manceuve « linéaire », cette dernière est définie par la vitesse maximum du liquide dans la conduite $V_{0}$ et le temps de fermeture complète correspondant Q6 o. Nous admettrons que, pour un degré d'ouverture $n$ différent de la pleine ouverture $n_{0}=1$, on a :

$$
V=n_{0} V_{0} \quad 0=n_{0} \theta_{0}
$$

d'où :

$$
\rho=\frac{a V}{2 g h_{0}}=n \rho_{0} \quad \frac{\rho}{0}=\frac{\rho_{0}}{\theta_{0}}==\mathrm{K}=\mathrm{C}^{\mathrm{te}}
$$

Dans ces formules, les indices 0 distinguent les parametres de pleine ouverture $\eta_{0}=1$ de ceux correspondant à une ouverture partielle $r_{i}$.

Le problème posé revient à étudier la variation des fonctions représentant le coup de bélier maximum lorsque $n=\frac{p 0}{i}$ varie de 0 à 1 . Son

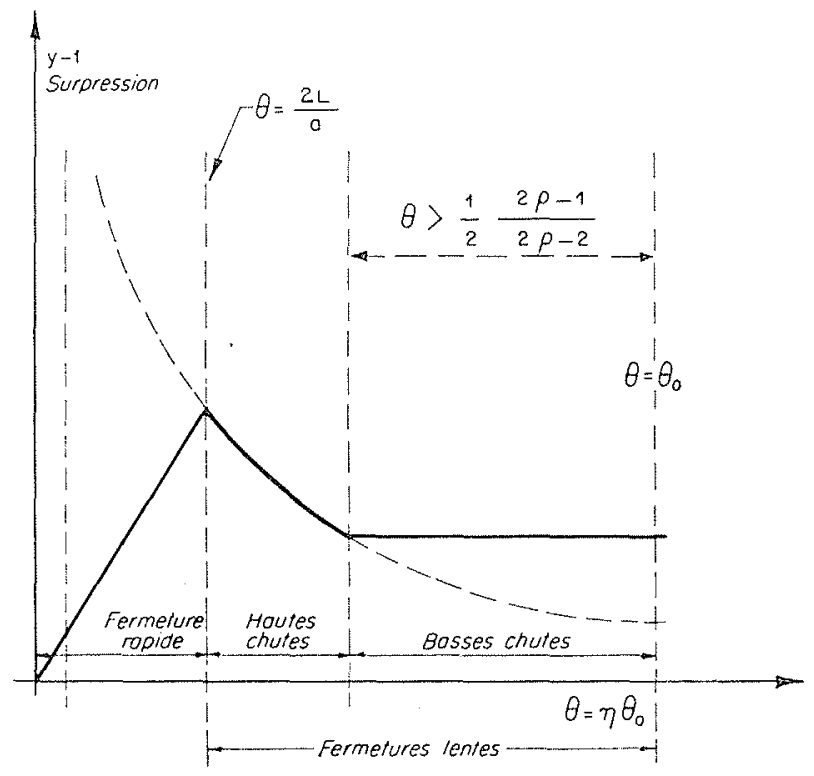

Fig. 7.

1. Cette hypothèse classique n'est pas tout à fait exacte lorsque l'obturateur est constitué par le distributeur d'une turbine hydraulique. La vitesse de fermeture de cet orane est sensiblement constante au cours d'une même manouvre, mais prend une valeur d'autant plus faible que le degré d'ouverture initial $\eta$ est plus petit. (Voir AmManet : Du coup de bélier maximum au cours des manouvres de fermeture d'une turbine hydraulique par son régulatteur de vitesse. Mull. Charmilles, n" 1 , mai 1945.) étude conduit au diagramme bien connu de M. Gariel (fig. 7) qui montre que pour $\theta_{0}>1$ (c'est-à-dire lorsque le temps de fermeture complète à partir de la pleine ouverture correspond à une manouve lente) le coup de bélier maximum se produit pour une fermeture partielle à partir d'une ouverture $\eta_{1}$ telle que $\eta_{0}=1$ (soit $\left.\mathscr{G}=\frac{2 \mathrm{~L}}{a}\right)$; sa valeur est donnée par la vieille formule de Mrchaud :

$$
h-h_{0}=\frac{2 \mathrm{~L} \mathrm{~V}_{0}}{g \mathscr{G}_{0}}
$$

Au contraire, dans le cas des fermetures rapides $\left(\theta_{0}<1\right)$, la valeur maximum possible du coup de bélier correspond toujours à une fermeture complète à partir de la pleine ouverture et a pour cxpression :

$$
h-h_{0}=\frac{a \mathrm{~V}_{0}}{g \widetilde{\mho}_{3}}
$$

b) INFLUENCE D'UNe RÉDUCTION DE LA CÉLÉRITÉ $a$ :

Dans le cas d'une fermeture rapide, toute réduction de célérité diminue dans les mêmes proportions la surpression maximum possible. Dans celui d'une fermeture lente, il est clair, d'après ce qui précède, que pour qu'une réduction de la célérité dans le rapport $\alpha=\frac{a}{a^{\prime}}$ conduise à une diminution du coup de bélier « maximum possible »- donc de l'épaisseur de la conduite il faut qu'elle aboutisse, pour une même valeur de $\widetilde{\mho}_{0}$, à une fermeture rapide pour la conduite a célérité réduite; cela exige évidemment :

$$
\alpha>\theta_{0}
$$

c'est-à-dire que $\alpha=\frac{a}{a^{\prime}}$ soit supérieur au temps relatif de fermeture $\theta_{0}$ de linstallation.

Nous verrons que les dispositifs réducteurs de célérité que nous proposons donnent des valeurs de \% comprises entre 2 et 5 ; la condition ci-dessus peut donc être assez aisément satisfaite pour des conduites forcées de hautes et moyennes chutes, mais non pour celles de basses chutes qui correspondent généralement à des valeurs de $\theta_{0}$ plus élevées.

La diminution du coup de bélier « maximum possible» est égale à :

$$
\frac{V_{0}}{g \theta_{0}}\left(a-a^{\prime} \theta_{0}\right)
$$

elle est d'autant plus grande, pour $\mathrm{V}_{0}$ et $a$ donnés, que $a^{\prime}$ et $\theta_{0}$ seront plus petits. 


\section{III. - Répartition de la surpression maximum le long de la conduite.}

Dans les paragraphes I et II ci-dessus, nous avons étudié la surpression maximum en un point très particulier : l'extrémité aval de la conduite; pour déterminer les épaisseurs à donner à ses divers tronecons, il convient d'étudier maintenant la répartition de la surpression maximum le long de celle-ci.

\section{a) Fermetures linéaires rapides : 0 et $\theta^{\prime}<1$.}

La surpression atteint sa valeur maximum $\frac{a V_{0}}{g}$ à l'obturateur à linstant $\mathscr{O}$ (fin de la fermeture). Elle se propage vers l'amont en restant égale à elle-même (transmission intégrale) jusqu'au point $x=\mathrm{L}-\frac{a \mathscr{6}}{2}$ où elle rencontre l'onde négative réfléchie par le réservoir; à partir de ce point, elle décroît linéairement jusqu'au droit du réservoir.

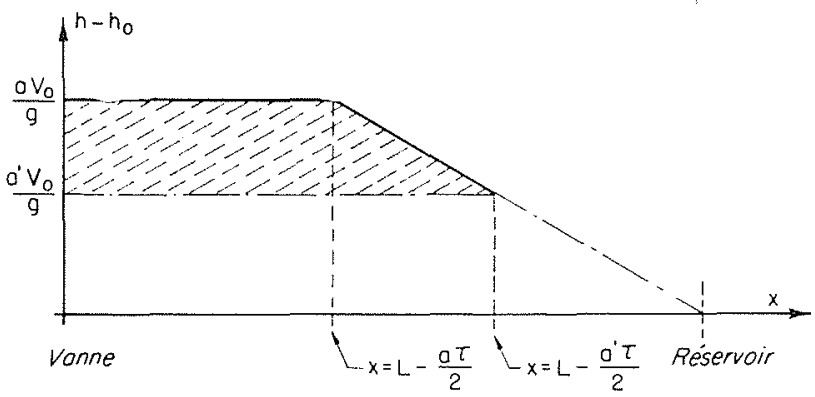

FIG. 8.

La figure 8 donne le diagramme de la répartition de la pression maximum le long de la conduite :

- En traits pleins pour une célérité $a$;

- En traits interrompus pour une célérité $a^{\prime}<a$.

Il est facile de voir que dans la zone « à répartition linéaire» les surpressions maxima sont les mèmes pour les deux conduites, tandis que dans la zone à « répartition intégrale » elles sont dans le rapport des célérités. L'économie réalisable sur le poids de la conduite, grâce au dispositif réducteur de célérité, est évidemment proportionnelle à l'aire hachurée de la figure 8 .

\section{b) Fermetures linéares lextes: 0 et $\theta^{\prime}>1$.}

On montre que si l'on porte les pressions maxima en différents points, calculées (comme il est dit plus haut) en fonction de la distance de ceux-ci à la vanne, on obtient très sensiblement une droite; on dit que la répartition des surpressions est linéaire. La réduction de la célérité de

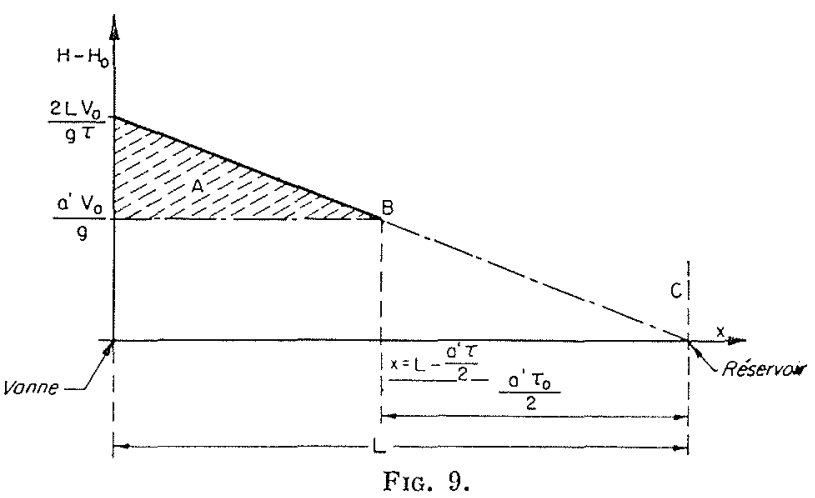

$a \grave{a} a^{\prime}=\frac{a}{\alpha}$ ne modifie en rien la répartition du coup de béliex maximum.

c) Cas ou la réduction de la CÉltérté transFORME LA FERMETURE LENTE EN FETMETURE RAPIDE : $0>1$ et $\theta^{\prime}<1$.

Les épaisseurs de la conduite classique $A$, de célérité $a$, sont alors déterminées par la répartition «linéaire » du coup de bélier de Mrchaud : $\frac{2 \mathrm{LV} V_{0}}{g \widetilde{\mathcal{O}}_{0}}$ (voir fig. 9). Pour la conduite $A^{\prime}$, de célérité $a^{\prime}$, on a une répartition « intégrale » $d u$ coup de bélier dans la partie basse de la conduite jusqu'au point d'abscisse $x=\mathrm{I}-\frac{a^{\prime} \mathscr{T}_{0}}{2}$. Audelà, la répartition est linéaire et telle que :

$$
h_{\mathrm{II}}-h_{0}=\frac{2 \mathrm{~V}_{0}(\mathrm{~L}-x)}{g \approx_{0}}
$$

Il est facile de voir que la droite qui repré-

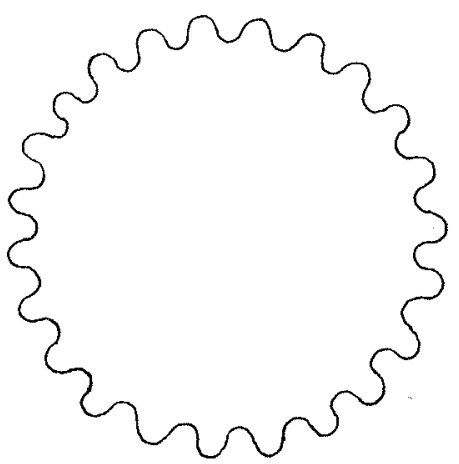

FIC. 10

sente cette équation coïncide avec celle relative à la répartition du coup de bélier de fermeture lente. 
L'économie réalisée sur le poids de la conduite par le dispositif réducteur de célérité est évidemment proportionnelle à l'aire hachurée sur la figure 9, on voit qu'elle est d'autant plus grande que $a^{\prime}$ est faible.

\section{IV. - Résumé et conclusion.}

Dans le cas de fermetures linéaires rapides, la réduction dans le rapport $\alpha$ de la célérité des ondes élastiques dans une conduite réduit toujours dans le même rapport $\approx$ le coup de bélier maximum.

Pour les fermetures linéaires lentes, la réduction de la célérité peut, suivant le cas, augmenter, diminuer ou laisser inchangée la surpression maximum. Le coup de bélier «maximum possible» pour lequel doit être dimensionnée la conduite, est toujours diminué si a est plus grand que le temps relatif de fermeture $0_{0}$.

\section{REMARQUES :}

1. Pour simplifier l'exposé, nous n'avons considéré, dans le cadre de la théorie d'Alrnevr, que le cas des manœuvres linéaires. Les cas plus complexes pourront toujours être étudiés par la méthode graphique de M. Bergeron.

2. L'étude des manœuvres d'ouverture donnerait lieu à des développements parallèles à ceux donnés plus haut pour les fermetures, sous réserve de tenir compte éventuellement des phénomènes de cavitation, souvent dangereux dans le cas de manœuvres rapides.

3. Nous avons supposé que le temps de fermeture "̈̈ était le même pour les conduites de célérité $a$ et $a^{\prime}$; la réduction de célérité entraîne généralement alors une diminution de la surpression maximum et, par suite, du poids de la conduite. Dans le cas d'une conduite forcée d'usine hydro-électricue on peut, en variante, réduire le temps de fermeture ó de la conduite de célérité $a^{\prime}$ jusqu'à obtenir la même valeur du coup de bélier que dans la conduite classique et rechercher une économie dans la réduction du $\mathrm{PD}^{2}$ indispensable du groupe turbine-alternateur, pour limiter la survitesse à une valeur donnée.

\section{DISPOSITIFS PERMETTANT LA RÉDUCTION DE LA CÉLÉRITÉ DES ONDES ELASTIQUES DANS UNE CONDUITE}

\section{I. - Divers moyens susceptibles d'être envisagés pour réduire la célérité $a$ :}

La célérité des ondes élastiques dans une conduite est donnée par la formule d'Alliévi :

$$
\frac{1}{a^{2}}=\frac{\Phi}{g}\left(\frac{1}{\varepsilon}+\frac{\mathrm{D}}{\mathrm{E} e}\right)
$$

On montre facilement que :

- le terme $\frac{1}{\varepsilon}$ représente la déformation cubique relative du liquide;

- le terme $\frac{\mathrm{D}}{\mathrm{E} e}$ la déformation relative $2 i_{2}$ de l'aire de la section mouillée de la conduite

\author{
sous l'action d'une surpression égale d \\ l'unité 1 .
}

Pour abaisser la célérité $a$, il faut s'efforcer d'augmenter par des artifices chacun de ces deux termes.

1. En négligeant la déformation longitudinale de la conduite la formule d'ALLiev coüncide avec celle plus générale de Kontewer, que l'on écrit sous la forme:

$$
\frac{1}{a^{2}}=\frac{\bar{\sigma}}{g}\left(\frac{1}{\varepsilon_{1}}+\frac{1}{\varepsilon_{2}}\right)
$$

$\varepsilon_{1}$ et $\varepsilon_{2}$ sont des pressions définies par les relations :

$$
\frac{\Delta_{1} w}{w}=\frac{\Delta p}{\varepsilon_{1}} \quad \text { et } \quad, \frac{\Delta_{2} w}{w}=\frac{\Delta p}{\varepsilon_{2}}
$$

dans lesquelles $\Delta_{1} w$ est l'augmentation du volume de la conduite et $\Delta_{2} w$ la diminution du volume du liquide, provoquées par l'augmentation de pression $\Delta p$. 
Le terme $\frac{1}{\varepsilon}$ est fixé par la nature du fiuide à transporter. Dans le cas d'un liquide, on pourrait augmenter sa valeur en mélangeant à celui-ci, d'une facon très homogéne, un fluide plus compressible - l'air par exemple; - pour des raisons techniques évidentes, cela serait, en génćral, peu pratique. Cette remarque explique pourtant que, dans certaines conduites transportant de l'eau plus ou moins émulsionnée d'air accidentellement, on ait mesuré des célérités bien inférieures à celles données par les formules théoriques.

Le deuxième terme $2 i_{2}$ semble plus facite à modifier. On peut tout d'abord constituer la paroi de la conduite par un matériau ayant un module d'élasticité très inférieur à celui de l'acier: alliage d'aluminium, caoutchouc plus ou moins armé par de la toile ou de l'acier, ma- tières plastiques, etc...; c'est là, vraisemblablement, une solution coùteuse et de mise au point assez longue en égard à la rusticité et à la robustesse que doit conserver la conduite.

Une autre formule consisterait à adopter, pour la section droite des conduites, non plus un cercle, mais une figure donnant, sous l'action des surpressions, des flèches de parois importanles, tout en conservant un rayon hydraulique acceptable; on pourrait envisager par exemple des conduites réalisées par roulage d'une tôle ondulée usuelle, comme l'indique la figure 10, ou encore des sections monillées en forme de losange, d'ellipse oli bien constituée par 2 segments de cercle égaux accolés par leur corde. Sauf cas particulier, toutes ces dispositions semblent n'avoir qu'un intérêt théorique.

Il sera en général plus pratique d'adopter le système suivant (fig. 11) : à l'intérieur et sur

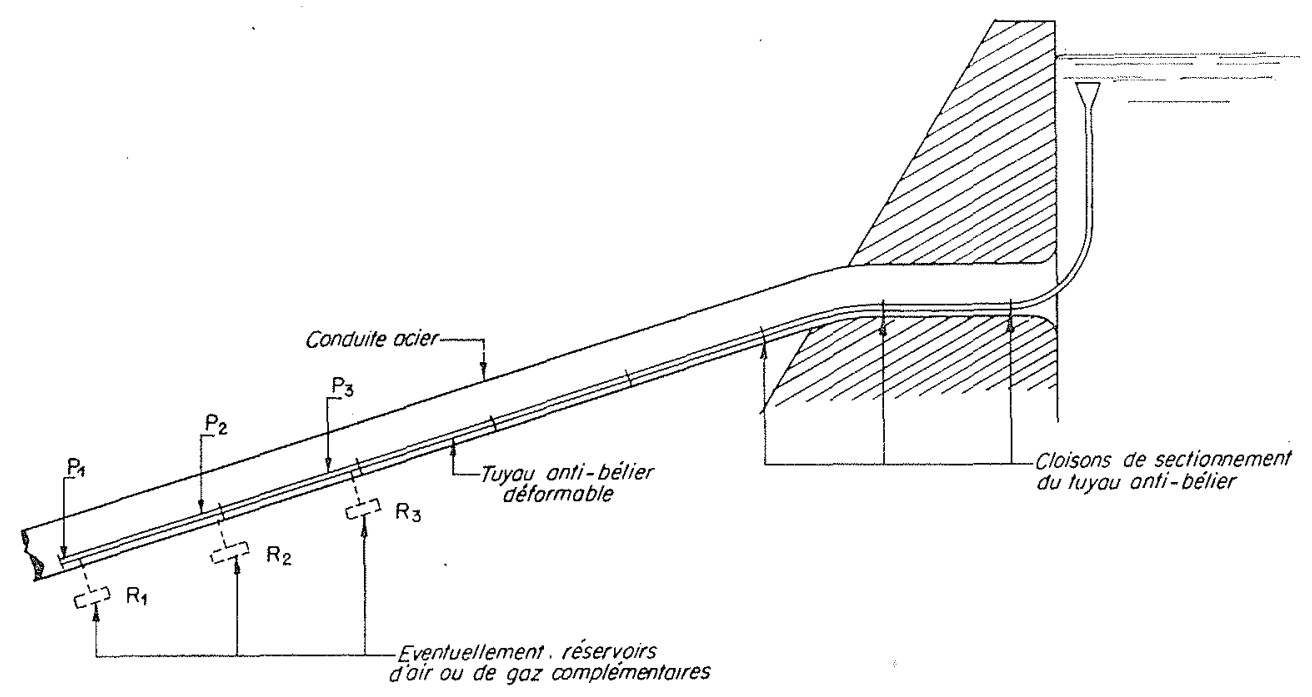

Fic. 11.

toute la longueur d'une conduite rigide classique (en acier, en béton armé, etc...) on dispose, suivant une génératrice, un élément élastique dont la déformation relative est très grande par rapport à celle de la paroi de la conduite el du liquide qu'elle contient. Cet élément élastique. peut être constitué par exemple par un tuyau à paroi mince et souple en caoutchouc ou en métal, contenant de l'air (ou un autre gaz ou vapeur plus compressible et éventuellement liquéfiable) dont la pression reste à chaque instant égale à celle du liquide qui l'entoure par le libre jeu de la déformation dudit tuyau. On concoit qu'en raison de son élasticité beaucoup plus grande que celle du liquide et de la paroi de la conduite l'élément antibélier ainsi réalisé puisse, avec un faible diamètre, augmenter sensiblement la déformation relative $2 i_{2}$ de la sec- tion mouillée et diminuer corrélativement la célérité a dans la conduite.

Même en régime permanent, la pression dans la conduite varie en général sensiblement d'un point à un autre, du fait des dénivellations de son profil en long et des pertes de charge; il est donc nécessaire de "sectionner» le tuyau antibélier en divers tronçons autonomes, de longueur telle, que les pressions hydrauliques au droit de leurs extrémités ne soient pas trop différentes.

On peut tenter d'éviter cette complication en utilisant non plus les variations de volume d'un gaz, mais celles d'un solide très déformable tel que le liège ou le caoutohouc. L'élément élastique antibélier serait alors constitué soit par un cordon cylindrique plein, soit par un tuyau à parois relativement épaisses. Si l'on emploie ce 
dernier dispositif, le tuyau devra être fermé à l'extrémité aval; l'extrémité amont pourra soit être fermée, soit être mise en communication avec le réservoir en charge qui alimente la conduite; ainsi, dans chaque section droite du tuyau antibélier, la pression du liquide est sensiblement égale à la pression régnant dans la conduite principale en régime permanent; elle doit, en principe, conserver cette même valeur lorsque la pression dans cette dernière est modifiée par le coup de bélier, de facon à mettre en jeu l'élasticité de la seule paroi du tuyau antibélier (un certain nombre de diaphragmes percés d'un très petit orifice seraient disposés dans ce dernier pour empêcher pratiquement la propagation du comp de bélier dans le liquide).

Ainsi que nous avons pu le vérifier par l'expérience, les caoutchoucs industriels normaux ne peuvent être utilisés pour constituer un tel élément antibélier car, sous l'action de la pression, ils se déforment en conservant leur volume (leur coefficient de Poisson est presque exactement égal à 0,5). Certains caoutchoucs « chargés» de liège pulvérisé ou des caoutchoucs ou des ébonites «mousse» spéciaux pourraient peut-être résoudre le problème ${ }^{1}$.

Les dispositions de détail de l'élément antibélies ne pourront guère être mises au point que par l'expérience.

Pour fixer les idées sur l'efficacité de pareils systèmes, nous allons calculer une valeur approchée de la célérité a par un dispositif très schématique utilisant uniquement la compressibilité de l'air.

\section{II. - Tuyau anti-bélier utilisant uniquement la compressibilité de l'air.}

\section{a) Description du prspositif :}

A l'intérieur, et sur toute la longueur d'une conduite métallique de diamètre $D$ et d'épaisseur $e$, nous disposons, suivant la génératrice inférieure par exemple, un tuyau de section $s_{0}$ rempli d'air comprimé dont nous supposons la paroi très mince et constituée de telle facon que sa tension reste toujours négligeable vis-à-vis des pressions en jeu.

Le tuyau antibélier est cloisonné de manière à constituer $n$ (fig. 11) réservoirs d'air dont la pression se met instantanément en équilibre avec la moyenne de celles de l'eau qui l'entoure; les cloisons transversales sont fixes, de sorte que

1. A noter que cela revient à utiliser la compressibi. lité de lair contenù dans les cellules du liège ou du caontchouc mousse. les variations de pression de l'air se répercutent uniquement sur le diametre $d$ du tuyau. Ce dispositif schématique équivaut à une série de bulles d'air formant un tube continu au sein du liquide, mais on concentre sur le diamètre du tube la déformation due aux variations de pressions de l'air. Nous négligerons la propagation des pressions par onde dans le tuyau de caoutchouc et dans lair, ce qui est légitime si les cloisons transversales sont assez voisines. Dans le cas contraire, on pourra disposer entre les cloisons des diaphragmes percés d'un petit orifice; les pertes d'énergie engendrées par ces derniers contribueront à l'amortissement du coup de bélier.

Il semble que l'on puisse se rapprocher, en pratique, de ce schéma en utilisant un tuyau de caoutchouc ou de métal ${ }^{1}$ à paroi mince ondulée suivant les génératrices (fig. 10), sectionné par des cloisons rigides maintenues à distance constante, par exemple par des armatures métalliques disposées suivant les génératrices.

Il n'est pas sûr que la section circulaire soit celle qui donnera les meilleurs résultats; il peut y avoir intérêt à favoriser le «flambage des parois du tube antibélier en donnant, à sa section droite, la forme d'un rectangle allongé, ou encore l'une de celles envisagées page 180.

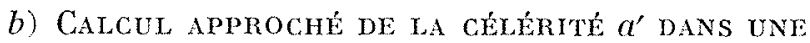
CONDUITE MUNIE D'UN TUYAU ANTT-BÉLIER A COMPRESSION D'AIR :

Pour déterminer la célérité $a^{\prime}$, nous calculerons tout d'abord :

1. La dilatation cubique relative du liquide;

2 La déformation relative $2 i_{2}$ de l'aire de la section nette de passage de l'eau sous l'action d'une surpression $p$.

La célérité $a^{\prime}$ s'en déduira en portant ces valeurs dans la formule:

$$
\frac{p}{\frac{\pi}{g} a^{2}}=j+2 i_{2}
$$

qui résulte directement de la formule (1) d'ALLYÉVI.

1) Calcul de $j$ :

Par définition, on a :

$$
j=\frac{p}{\varepsilon i}
$$

1. Construction un peu analogue à celle des capsules manométriques ntilisées dans certains appareils de mesure on de réglagge automatique. 


\section{2) Calcul de 2 i2:}

La déformation absolue de laire occupée par l'eau sous l'action de la surpression $p$ est la somme de deux termes qui s'ajoutent :

$1^{\circ}$ La déformation absolue $2 i_{2}^{\prime} \frac{\pi \mathrm{D}^{2}}{4}$ de la conduite en acier avec :

soit en posant :

$$
i_{2}^{\prime}=\frac{\mathrm{N}}{\mathrm{E}}=\frac{p \mathrm{D}}{2 e \mathrm{E}}
$$

$$
\begin{gathered}
\frac{\pi \mathrm{D}^{2}}{4}=\mathrm{S}_{0} \\
2 i_{2}^{\prime} \mathrm{S}_{0}=\frac{p \mathrm{D}}{e \mathrm{E}} \mathrm{S}_{0}
\end{gathered}
$$

$2^{\circ}$ La déformation absolue $2 i^{\prime} .2 s_{0}$ du tuyau antibélier rempli d'air comprimé.

Nous allons calculer cette dernière dans l'hypothèse où la compression de l'air étant isotherme et la longueur du tuyau entre deux cloisons invariable, la déformation du tuyau antibélier porte uniquement sur sa section droite $s_{0}$. On a alors, si $P_{0}$ est la pression absolue initiale de l'air comprimé et $s_{1}$ la section droite du tuyau antibélier lorsqu'il est soumis à la pression $\mathrm{P}_{0}+p$ :

d'où :

$$
\mathrm{P}_{0} s_{0}=\left(\mathrm{P}_{0}+p\right) s_{1}
$$

$$
\begin{aligned}
& \frac{p}{\mathrm{P}_{0}}=-\frac{s_{1}-s_{0}}{s_{1}} \\
& 2 i^{\prime \prime}{ }_{2}=\frac{s_{1}-s_{0}}{s_{0}} ;
\end{aligned}
$$

si $s_{1}$ n'est pas très différent de $s_{0}$, on peut donc admettre en première approximation :

$$
2 i_{2}^{\prime \prime} s_{0}=-\frac{p}{\mathrm{P}_{0}} s_{0}
$$

Au total, la déformation relative $2 i_{2}$ de l'aire de la section effective de passage du liquide dans la conduite $\left(\Sigma_{0}=\mathrm{S}_{0}-s_{0}\right)$ sous l'action de la surpression $p$ sera :

$$
2 i_{2}=\frac{\mathrm{s}_{0} \frac{p \mathrm{D}}{e \mathrm{E}}+\mathrm{s}_{0} \frac{p}{\mathrm{P}_{0}}}{\Sigma_{0}}
$$

d'où, puisque :

$$
\begin{gathered}
\frac{p}{\frac{\omega}{g} a^{2}}=j+2 i_{2} \\
\frac{1}{a^{2}}=\frac{\pi}{g}\left[\frac{1}{\varepsilon}+\frac{S_{0}}{\Sigma_{0}} \frac{1}{\mathrm{E}} \frac{\mathrm{D}}{e}+\frac{s_{0}}{\Sigma_{0}} \frac{1}{p_{0}}\right]
\end{gathered}
$$

Pour les applications numériques, en prenant comme unité le mètre, le kilogramme et la seconde, et en posant :

$$
\begin{array}{r}
k=\frac{10^{10}}{\mathrm{E}} \quad \text { (Alditévi) } \\
\frac{s_{0}}{\Sigma_{0}}=c, \text { d'où } \frac{\mathrm{S}_{0}}{\Sigma_{0}}=\frac{\Sigma_{0}+s_{0}}{\Sigma_{0}}=1+c
\end{array}
$$

on peut mettre cette formule sous la forme :

$$
\frac{1}{a^{2}}=\frac{\omega}{g}\left[\frac{1}{\varepsilon}+(1+c) k \frac{\mathrm{D}}{e}+10^{1 \frac{c}{\mathrm{P}_{0}}}\right]
$$

Dans le cas particulier d'une conduite parcourue par de l'eau, cette dernière formule devient :

$$
a=\frac{9.900}{\left[48,3+(1+c) k \frac{\mathrm{D}}{e}+\frac{c}{\mathrm{P}_{0}} \times 10^{10}\right]^{1 / 2}}
$$

Comparons cette expression à celle de la classique formule d'Allívi :

$$
a=\frac{9.900}{\left[48,3+k \frac{\mathrm{D}}{e}\right]^{1 / 2}}
$$

On voit que l'adjonction de l'élément antibélier se traduit par l'addition à l'expression entre parenthèses au dénominateur de deux termes :

- L'un, ck $\frac{\mathrm{D}}{e}$ tient compte de ce que la dilatation absolue de la paroi en acier restant la même, la dilatation transversale « relative » de l'eau est plus grande puisqu'elle s'applique à une aire effective $\left(\Sigma_{0}=\mathrm{S}_{0}-s_{0}\right)$ plus petite. Ce terme sera le plus souvent négligeable;

- L'autre, $\frac{c}{\mathrm{P}_{0}} \times 10^{10}$, traduit la compressibilité de l'air enfermé dans le tuyau antibélier.

L'abaque de la figure 11 bis donne les valeurs de la célérité $a^{\prime}$ et du rapport $\alpha=\frac{a}{a^{\prime}}$ en fonction de la pression de service $\mathrm{H}_{0}$ en mètres d'eau et du rapport $\mathrm{C}=100 \frac{s_{0}}{\mathrm{~S}_{0}-s_{0}}$ pour des conduites d'eau en acier calculées pour un taux de travail de $10 \mathrm{~kg} / \mathrm{mm}^{2}$; on a supposé $\mathrm{P}_{0} \# \mathrm{H}_{0}+10 \mathrm{~m}$ d'eau. On voit que pour $\mathrm{C}=1$ la réduction. de célérité est de l'ordre de 2,7 pour $\mathrm{H}_{0}=100 \mathrm{~m}$ et de 1,6 pour $\mathrm{H}_{0}=1.000 \mathrm{~m}$; ces valeurs de a passent respectivement à 5,8 et à 3,1 pour $\mathrm{C}=\mathbf{5}$. Pour des conduites on des galeries en béton on pour des conduites en acier ayant un taux de travail inférieur au chiffre de $10 \mathrm{~kg} / \mathrm{mm}^{2}$ admis 


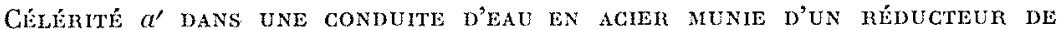
CÉLÉRITÉ A COMPRESSION D'AIR :

$$
a^{\prime} \# \frac{9.900}{\left[48,3+\frac{10^{4}}{\mathrm{H}_{0}}+\frac{10^{3} \mathrm{C}}{\mathrm{H}_{0}+10}\right]^{1 / 2}}
$$

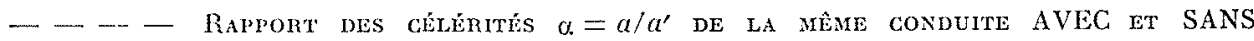
RÉDUCTEUR DE GÉLÉRITÉ

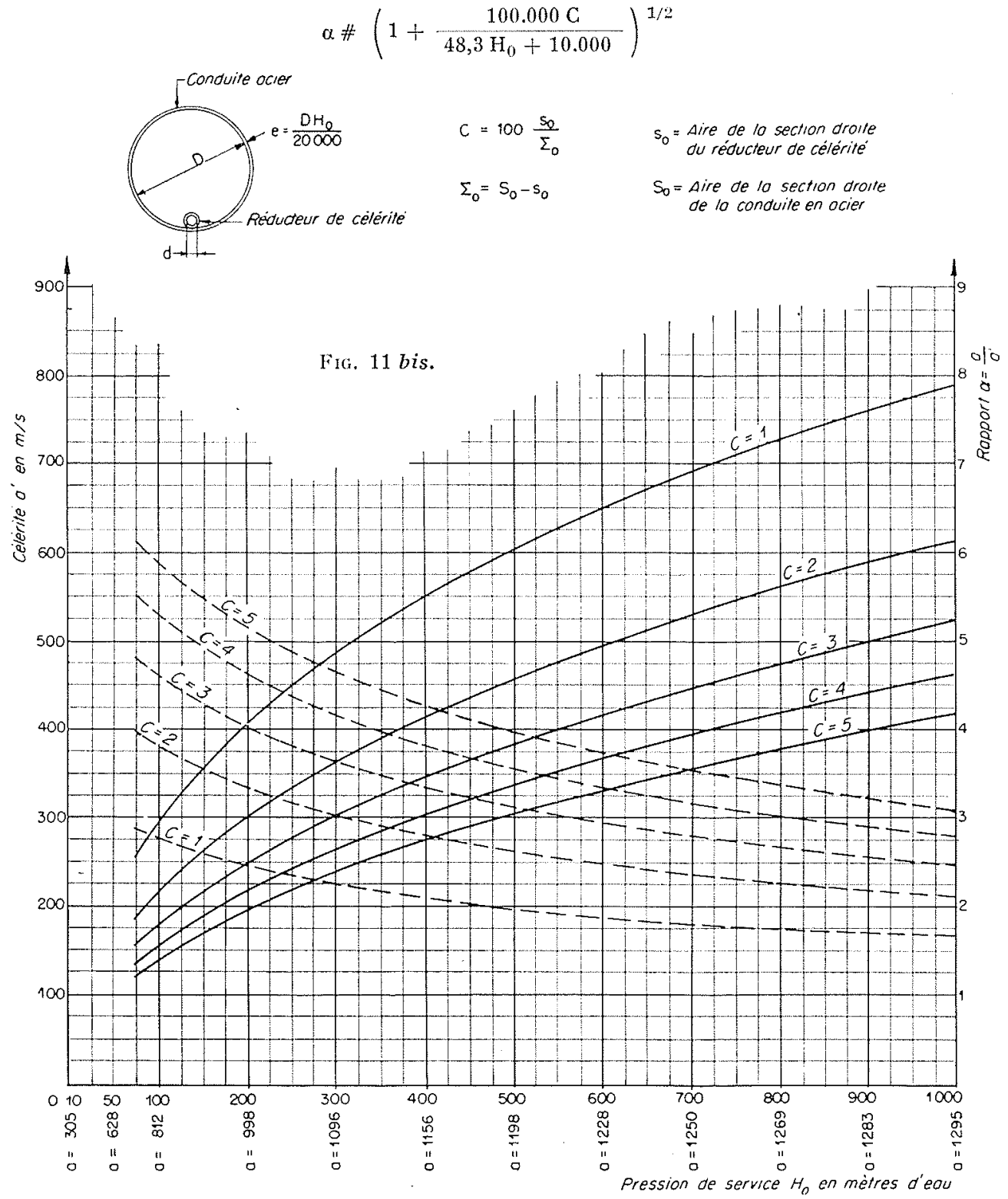

dans notre abaque, la réduction de célérité obtenue serait, toutes choses égales d'ailleurs, plus importante.

La célérité est une fonction croissante de la pression $\mathrm{P}_{0}$ de l'air comprimé; or, celte pression, sensiblement égale à celle existant dans la conduite en régime permanent, sera en général différente d'un point à un autre (dans le cas d'une conduite forcée inclinée notamment). II en résulte que la célérité variera d'un point à l'au- tre de la conduite qui ne sera plus à « caractéristique unique * (à moins que par construction on conserve constant le rapport $\frac{c}{\mathrm{P}_{0}}$, ce qui sera. souvent difficile).

Les formules données au chapitre premier cidessus donneront souvent alórs une approximalion insuffisante, mais la méthode graphique de M. Bergerox peut être adaptée sans grande difficulté à l'étude des conduites à célérité variable. 


\section{VÉRIFICATION EXPÉRIMENTALE DE L'EFFICACITÉ DU RÉDUCTEUR DE CÉLERITE A COMPRESSION D'AIR}

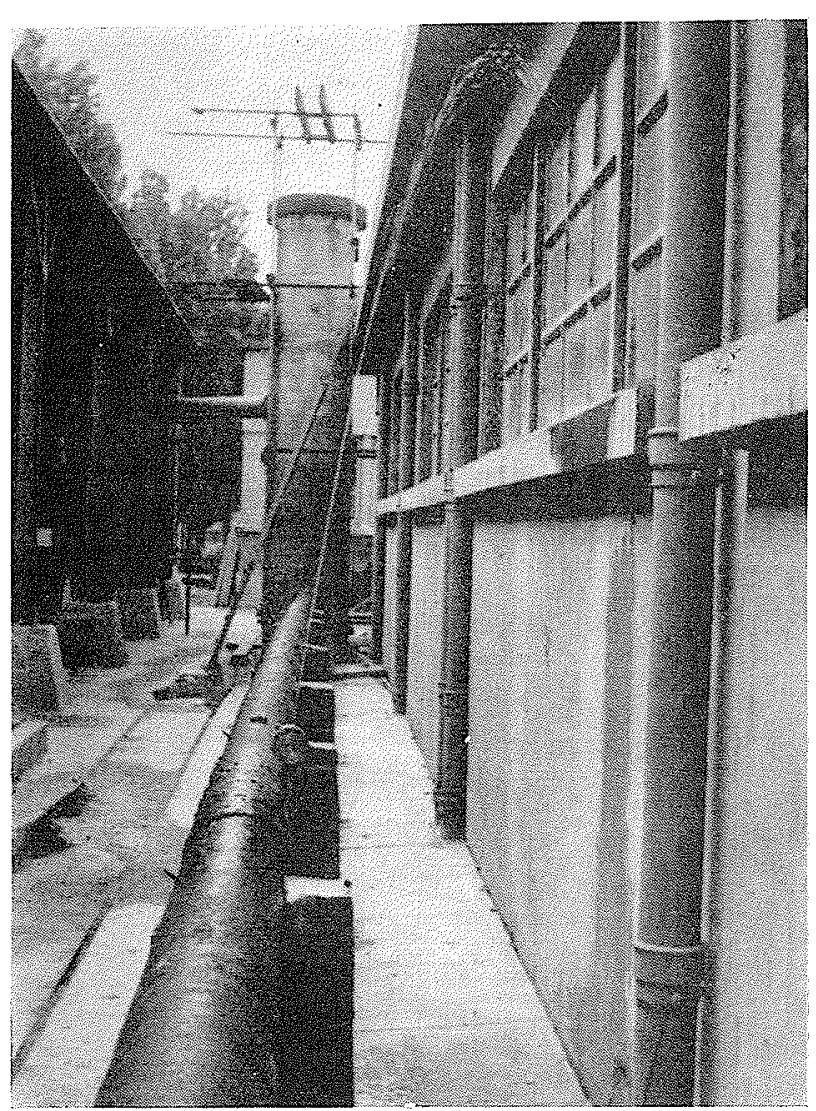

FrG. 12.

Condutte et CHEMinée d’éQuilibre.

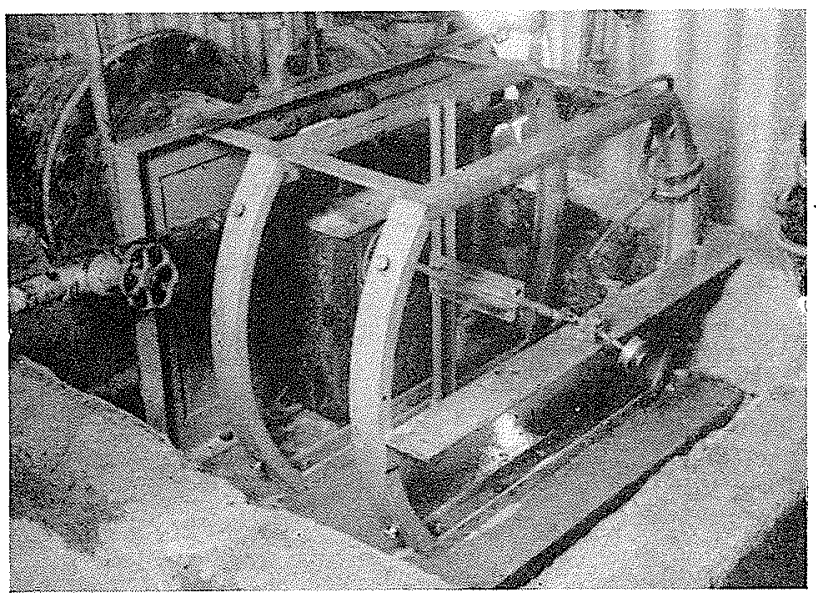

FIG. 13.

DISPOSITHF DE FERMETURE RAP'DE.
Nous avons pu procéder à quelques vérifications expérimentales des conceptions et des formules exposées dans le chapitre II en utilisant une conduite installée au Laboratoire national d'Hydraulique de Chatou, pour des mesures de pertes de charge; malheureusement, en raison des travaux d'extension du Laboraloire, nous n'avons pu disposer de cette installation pendant un temps suffisant pour procéder à des essais sytématiques.

Toutes les expériences relatées dans ce chapitre ont été effectuées par M. Literruer, Ingénieur au Service des Etudes et Recherches Hydrauliques de l'Electricité de France; nous lui renouvelons ici nos vifs remerciements pour sa précieuse collaboration.

\section{I. - Description de l'installation d'essai.}

\section{a) Conduite :}

La conduite utilisée est un pipe-line pour transport d'hydrocarbures; ses caractéristiques principales sont les suivantes:

Diamètres fextérieur..... $254 \mathrm{~mm}$

moyens. . intérieur..... D $\mathrm{D}=240 \mathrm{~mm}$

Epaisseur...........ee=7 mm (acier)

Section mouillée....... $\mathrm{s}_{0}=4,524 \mathrm{dm}^{2}$

La conduite est alimentée sous charge constante par le réseau de distribution d'eau du Laboratoire; le secteur d'essai, sensiblement horizontal (fig. 12), a une longueur de $90,50 \mathrm{~m}$ comptée depuis son origine (dans une chambre d'équilibre déversante de $0,90 \mathrm{~m}$ de diamètre intérieur) jusqu'à son extrémité aval, obturée par un clapet spécial permettant des fermetures pratiquement linéaires en quelques centièmes de seconde (fig. 13). La pression statique, sensiblement constante sur tout le secteur d'essai, - est de $5 \mathrm{~m}$ d'eau environ.

b) Dispositif réducteur de cÉlérité (fig. 14) :

Il est constitué par un tuyau de caoutchouc 


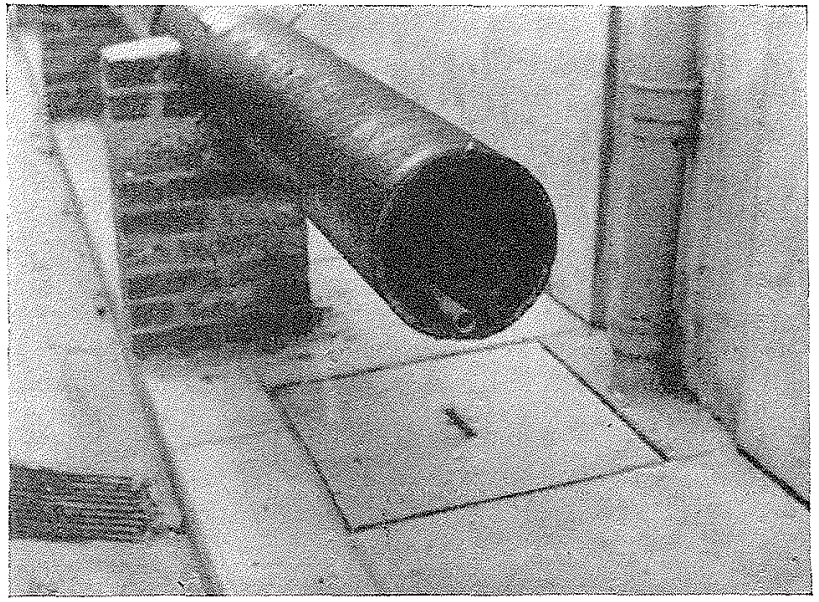

Fig. 14.

CONDUTE ET TUYAU DE CAOUTCHOUC, réducteur de célérité.

non entoilé du commerce ayant les caractéristiques ci-après :

$$
\begin{aligned}
& \text { Diamètres }\left\{\begin{array}{l}
\text { extérieur..... } \\
\text { intérieur..... } 28 \mathrm{~mm}
\end{array}\right. \\
& \text { Epaisseur...........e } e=2 \mathrm{~mm} \\
& \text { Section intérieure....... } s_{0}=4,524 \mathrm{~cm}^{2} \\
& c=\frac{s_{0}}{\mathrm{~S}_{0}-s_{0}}=\frac{1}{99}
\end{aligned}
$$

Ce tuyau est rempli d'air comprimé à une pression voisine de celle existant en régime permanent dans la conduite. L'expérience a montré que pour que la paroi du tuyau antibélier n'intervienne pas nolablement dans le phénomène, il fallait que la pression de l'air en régime permanent soit de l'ordre de $4,50 \mathrm{~m}$ d'eau; dans ces conditions, le tuyau «flambe» pour la moindre surpression et la tension élastique de la paroi de caoutchouc a une influence faible.

\section{c) EnRegistrement des surpressions :}

Les surpressions étaient enregistrées aux deux extrémités et vers le milieu du secteur d'essai par un manographe à strains-gage ${ }^{1}$ construit par le Laboratoire et soigneusement étalonné.

Les débits étaient mesurés soit par une capacité jaugée, soit par un petit déversoir taré au moyen de cette dernière.

1. La conception de cet appareil est due à M. VALEMwors, Ingenieur Chef de section au Laboratoire National d'Hydraulique.

\section{II. - Conduite des essais et résultats obtenus.}

Il a été exécuté deux séries principales d'essais consistant essentiellement dans le relevé, en fonction du temps -- aux trois points désignés ci-dessus -- de la surpression consécutive à des fermetures rapides du clapet aval pour différents débits. Dans la première série, la conduite ne comportait aucun dispositif particulier; dans la seconde, elle était munie du tuyau réducteur de célérité à compression d'air sommairement décrit plus haut.

Accessoirement, on a procédé, d'une part, à quelques essais de fermeture lente avec ce dernier dispositif et, d'autre part, vérifié qu'un tuyau de caoutchouc plein d'eau inséré dans la conduite n'entraînait aucune réduction sensible de célérité.

a) Essais sans réducteur de célértté :

La conduite ayant été soigneusement purgée d'air, il a été exécuté 19 essais avec des débits compris $^{1}$ entre 0,59 et $2,10 \mathrm{l} / \mathrm{s}$; la fermeture, suivant une loi sensiblement linéaire, du clapet se faisait en un temps voisin de $3 / 100^{\circ}$ de seconde, sensiblement inférieur à la durée de phase $\mu$ de la conduile qui est de l'ordre de $14 / 100^{\circ}$ de seconde.

La figure 15 donne, à titre d'exemple, le facsimilé d'un enregistrement relatif à la coupure rapide d'un débit de $1,15 \mathrm{l} / \mathrm{s}$; son allure générale est bien celle prévue par la théorie.

A partir d'un tel enregistrement, on peut calculer la célérité $a$, soit d'après l'amplitude de la «première surpression», soit en mesurant la période $\mathrm{T}=\frac{4 \mathrm{~L}}{a}$ de la courbe de surpression.

La première méthode est basée sur l'expression de la surpression maximum :

$$
h-h_{0}=\frac{a \mathrm{~V}_{0}}{g}
$$

d'où l'on tire :

$$
a=\frac{g\left(h-h_{0}\right)}{\mathrm{V}_{0}}
$$

1. Les faibJes vitesses correspondantes $(0,013$ a $0,045 \mathrm{~m} / \mathrm{s}$ ) sont imposées par la nécessité de ne pas créer de pressions négatives importantes, génératrices de cavitation lors du "contre-coup 》 de bélier, compte tenu de la pression statique de $5 \mathrm{~m}$ d'eau existant sur le tronçon d'essai. 
Essai №10

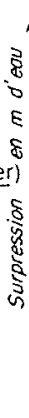

(5)

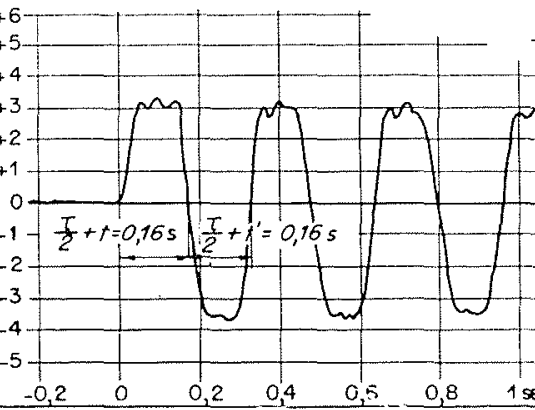

Surpression $\dot{a}$ lobturoteur

(sons dispositif onti-belier)

$Q=1,15 \mathrm{l} / \mathrm{sec}$

\#0.02 sec.

$\phi=240$

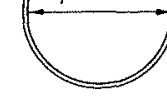

$-0,2$

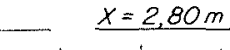 \\ - $\frac{x=2,80 m}{1}$}

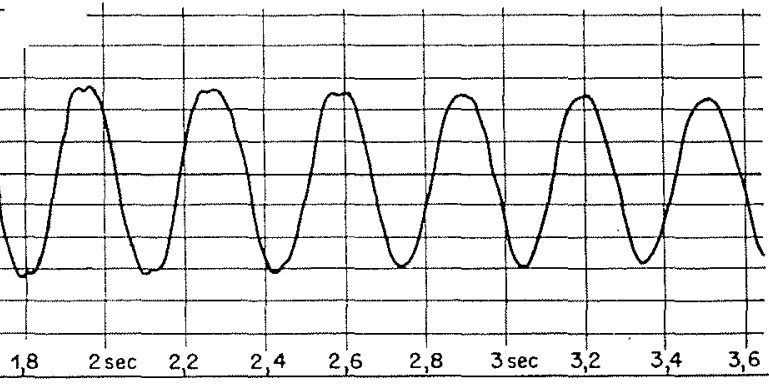

FiG. 15.

Essai №1 lavec dispositif onti-bélier (sans diaphrogme)

$\underline{Q}=7,65 \mathrm{l} / \mathrm{sec}$

Po $=14,85 \mathrm{~m}$.

$\tau$ 0,03 sec
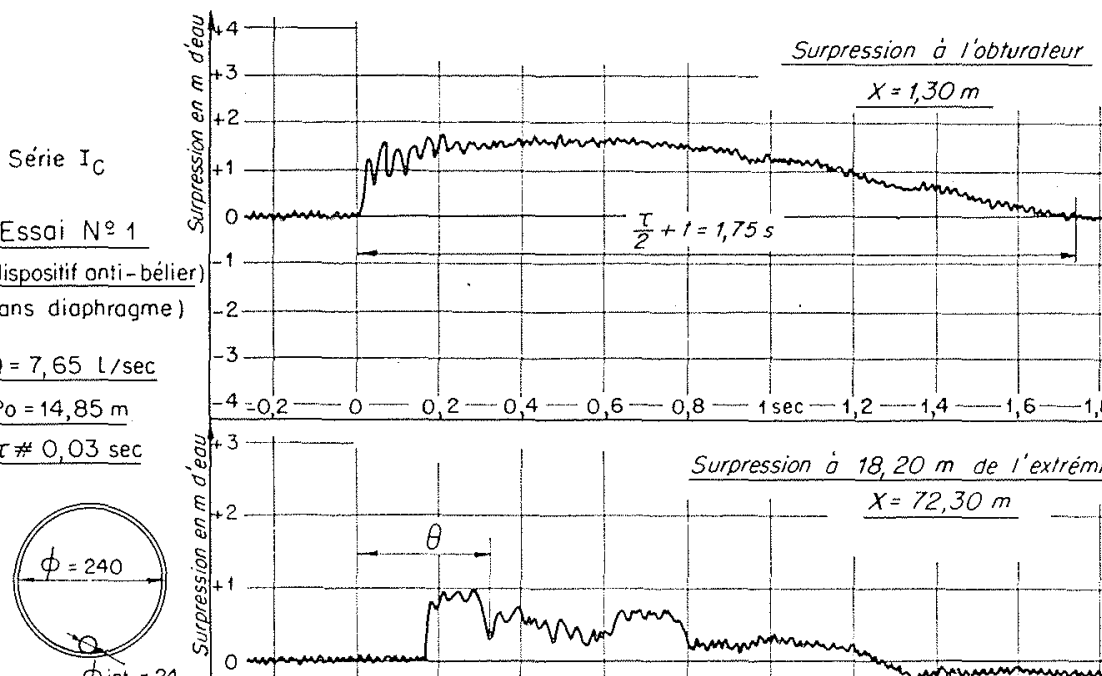

$-2$

$-3$

$-4=02-0+02-044$

Surpression $\dot{0} 18,20 \mathrm{~m}$ de l'extrémite amionl

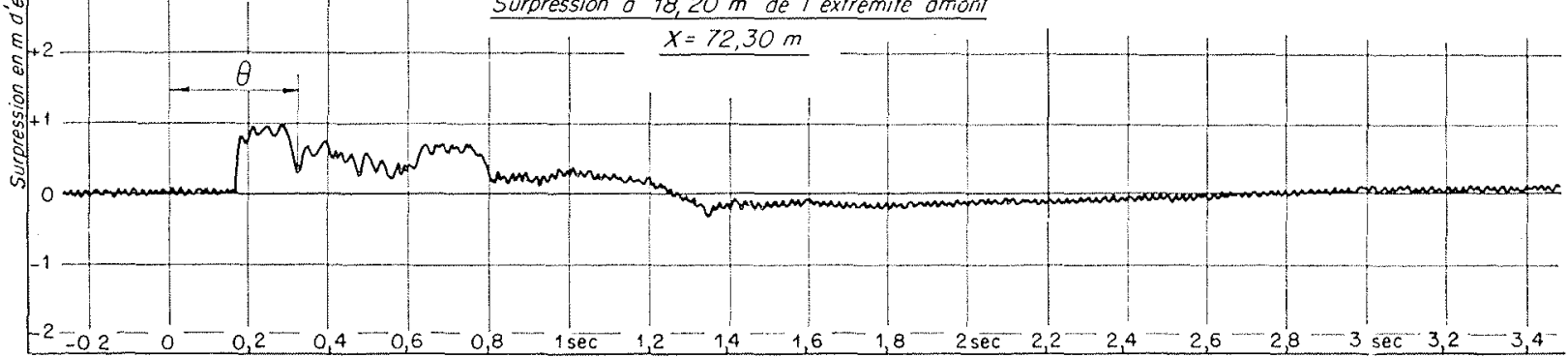

Fig. 19.

FIG. 16.

Fermeture rapioe.

Surpressions

en fonction du débit.

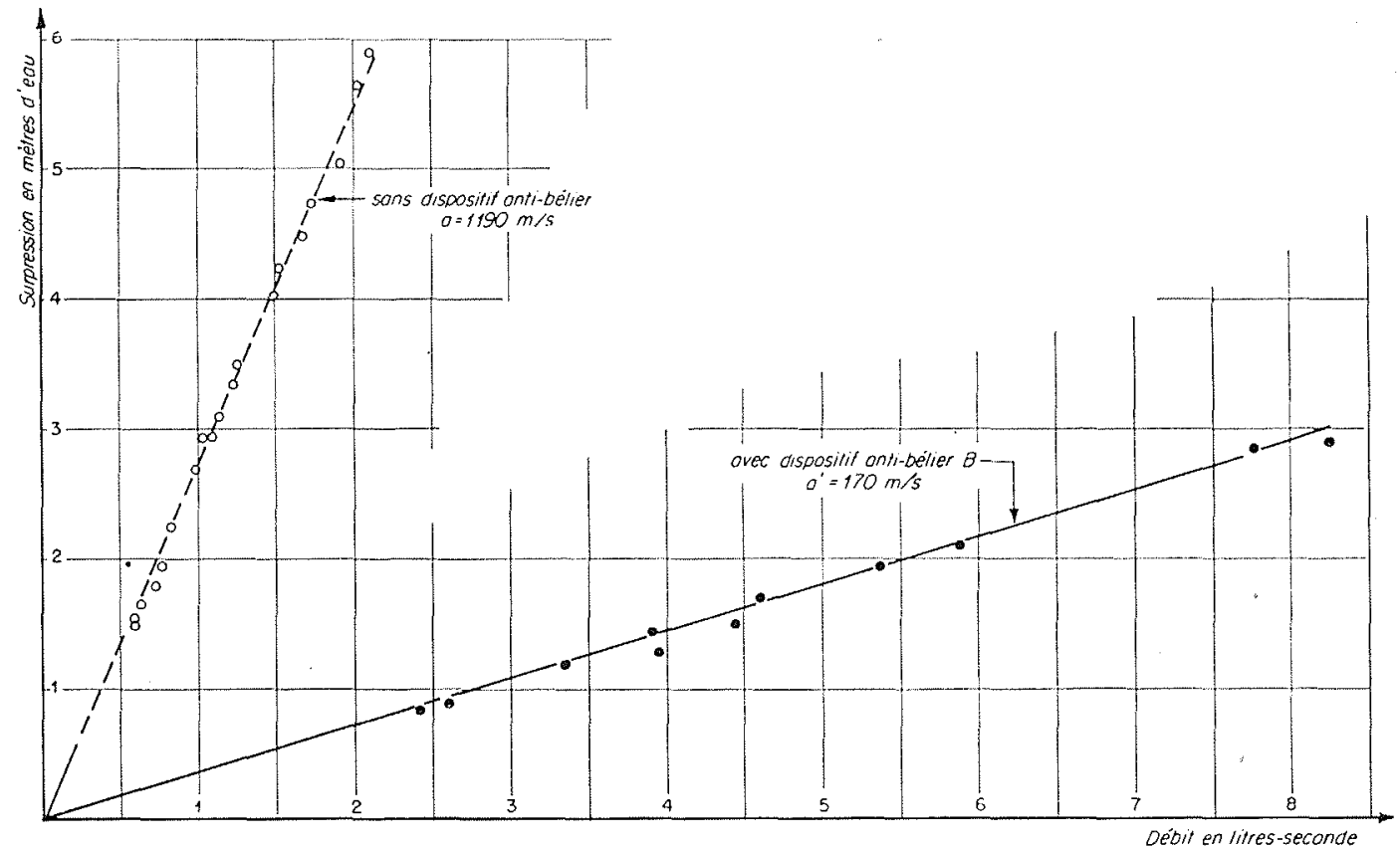


Pour ríaliser une certaine compensation des erreurs accidentelles, nous avons tracé (fig. 16) la courbe donnant les surpressions maxima mesurées en fonction des débits correspondants. Nous avons obtenu - ainsi que le prévoit la théorie -- une droite dont le coefficient angulaire est proportionnel à la célérité cherchée; celle-ci ressort à : $a_{h}=1.190 \mathrm{~m} / \mathrm{s}$.

D'autre part, la valeur moyenne de la période $\mathrm{T}=\frac{4 \mathrm{~L}}{a}$, mesurce sur les einq premières périodes, ressort pour les 19 essais à $0,305 \mathrm{~s}$, ce qui correspond à un cólérití $a_{1}=1.185 \mathrm{~m} / \mathrm{s}$. Compte tenu de l'approximation des mesures, les deux valeurs expérimentales de la cólérité $a^{h}$ et $a_{\mathrm{T}}$ sont pratiquement confondues; nous montrerons plus loin qu'elles sont en bonne concordance avec les valeurs calculées par les formules théoriques.

b) Essais avec réducteur de célérité a COMpRESSION D'AM (dit aNTHB́ler, type B) :

Il a été exécuté au total une quarantaine d'enregistrements de la surpression consécutive à la fermeture rapide de différents débits allant de 1,25 à $8,45 \mathrm{l} / \mathrm{s}$.

Nous nous sommes rapidement rendu compte que le fonctionnement du réducteur de célérité était assez fortement influencé par la tension mécanique de sa paroi de caoutchouc, laquelle dépend de la différence entre la pression $P$ de l'air contenu dans le tuyau et celle $\boldsymbol{H}$ de l'eau qui l'entoure. Schématiquement, on peut dire qu'il existe deux régimes principaux séparés par un régime de transition. Dans le premier, qui est réalisé dans nos essais, lorsque malgré le coup de bélier la pression absolue de l'eau reste toujours inférieure à celle de l'air augmentée de $0,5 \mathrm{~m}$ d'eau, la paroi du tuyau reste tendue et la contrainte du caoutchouc intervient dans le phénomène. Le deuxième correspond au cas où le tuyau est en permanence partiellement aplati par flambage, ce qui se produit, dans nos essais, lorsque la pression absolue de l'eau est supérieure de plus de $0,5 \mathrm{~m}$ d'eau à celle de l'air contenu dans le tuyau; dans ces conditions, Ia contrainte dans la paroi de caoutchouc intervient peu dans le phénomène, qui se rapproche de celui envisage dans les calculs théoriques du chapitre II. Enfin, le régime de transition survient lorsque, au cours de la surpression, la différence de pression entre les fluides contenus respectivement dans la conduite et dans le tuyau réducteur de célérité change de sens, de sorte que ce dernier est tantôt de forme circulaire, tantôt plus ou moins aplati par flambage de sa paroi.
1. Essais de la série $I_{A}$ et II correspondant au premier régime (paroi de caoutchouc tendue) :

(o) Réducteur de célérité sans diaphragmes (série $I_{\Lambda}$ )

La figure 17 donne à titre d'exemple les enregistrements correspondant à la «coupure» pratiquement linéaire en $3 / 100^{\circ}$ de seconde d'un débit de $4,32 \mathrm{l} / \mathrm{s}$, la pression absolue de l'air dans le tuyau réducteur de célérité étant de $16,30 \mathrm{~m}$ d'eau.

Considérons d'abord la courbe des surpressions au voisinage de l'obturateur (section d'abcisse $x=1,30 \mathrm{~m}$ comptée à partir de l'extrémité aval de la conduite). La première demipériode présente bien le «palier» de surpression classique des «fermelures rapides», mais celui-ci disparait rapidement au cours des autres périodes, pour faire place à une courbe d'allure sinusoïdale; il faut voir là, croyons-nous, l'influence d'un amortissement du coup de bélier beaucoup plus rapide que dans la conduite sans réducteur de célérité. On notera la présence de fluctuation de pression de fréquence beaucoup plus élevée que celle correspondant à la période propre $\frac{4 \mathrm{~L}}{\boldsymbol{a}}$ de la conduite; ces oscillations conduisent au début de la première demi-période à une «pointe» de pression qui rappelle 《l'onde de tête identifiée pour la première fois par BAzrn sur le front des longues intumescences dans les canaux à écoulement libre.

Tandis que le «palier» de la surpression conduirait à une célérité de l'ordre de $170 \mathrm{~m} / \mathrm{s}$, la «pointe» correspond à une célérité comprise entre 300 et $350 \mathrm{~m} / \mathrm{s}$, done très voisine de la vitesse de propagation d'un ébranlement dans l'air contenu dans le tuyau de caoutchouc entouré d'eau. L'enregistrement de la surpression dans l'air à l'extrémité du tuyau réducteur de célérité (troisième diagramme de la fig. 17) confirme d'ailleurs ces chiffres.

L'interprétation de cette série d'essais, suivant un schćma un peu grossier admettant la coexistence de deux ondes élastiques, l'une transmise par l'air dans le réducteur de célérité (qui «l'induit» ensuite dans l'eau), l'autre se propageant dans la conduite, semble justifiée par l'examen de la courbe de surpression relevée simultanément vers l'extrémité amont de la conduite $(x=72,30 \mathrm{~m})$.

En effet, on constate immédiatement qu'au cours de son trajet de l'aval vers l'amont, l'onde de surpression s'est considérablement distordue, au point que la première demi-période paraît presque scindée en deux parties. La célérité de la première surpression, estimée d'après le temps de parcours entre les sections $x=1,30 \mathrm{~m}$ et $x=72,30 \mathrm{~m}$ est de l'ordre de la vitesse du son dans lair enfermé dans le tuyau de caoutchouc, 


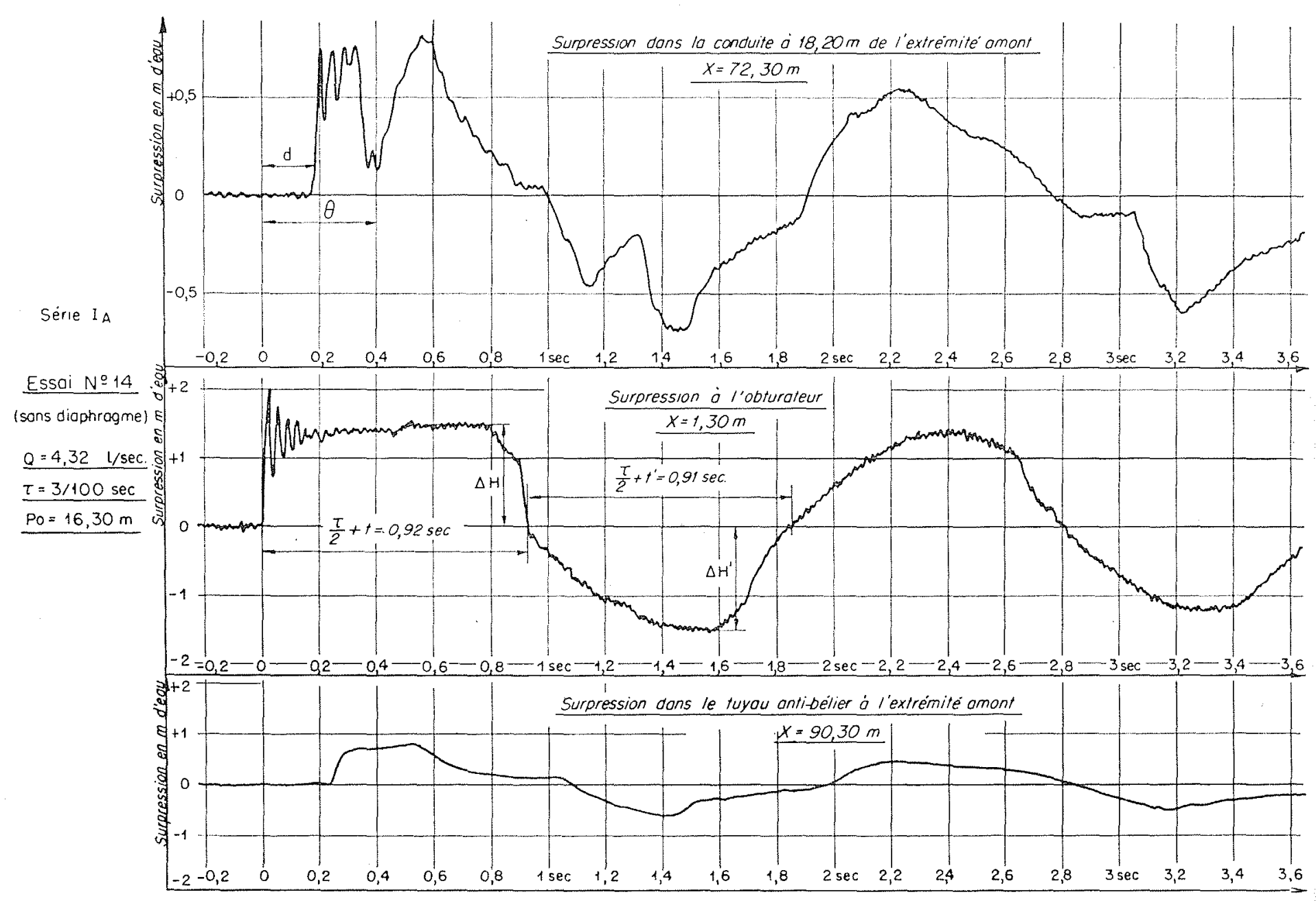

FIG. 17.

tandis que celle de la deuxième partic est voisine de la célérité de $170 \mathrm{~m} / \mathrm{s}$, correspondant à la surpression maximum au clapet-obturateur.

Nous avons reporté sur la figure 16 la droite «expérimentale» donnant l'amplitude du premier palier de surpression en fonction du débit «coupé» au cours de ces essais; le coefficient angulaire de cette droite conduit à assigner $\dot{a}$ la célérité $a^{\prime}$ ane valeur constante de $170 \mathrm{~m} / \mathrm{s}$, soit 14\% environ de la célérité dans la mème conduite non munie du réducteur de célérité.

\section{B) Réducteur de célérité muni de diaphragmes (série $11 \mathrm{~A}_{\mathrm{A}}$ ):}

Pour éviter lá transmission des surpressions par l'air contenu à l'intérieur du tuyau réducteur de célérité, nous avons intercalé sur la longueur de ce dernier neuf diaphragmes en laiton équidistants, percés chacun d'un trou de $1 \mathrm{~mm}$ de diamètre. Par le jeu des réflexions sucessives des ondes sur ces diaphragmes et, par suite, des pertes d'énergie dues aux orifices qu'ils comportent, nous espérions ainsi réduire les «pointes» et augmenter l'amortissement.

C'est bien ce qu'ont montré les essais de la série $I_{A}$ dont la figure 18 reproduit, à titre d'exemple, un enregistrement-type. A l'obturateur, la «pointe» du début de la première surpression est presque entièrement éliminée et les fluctuations de pression à haute fréquence sont très diminuées. La comparaison des courbes de surpressions aux abcisses $x=1,30 \mathrm{~m}$ et $x=$ $72,30 \mathrm{~m}$ ne permet plus de distinguer la propagation de deux ondes distinctes comme dans les diagrammes de la figure 17. Le coup de bélier dans l'air du tuyau réducteur de célérité est en synchronisme avec celui existant dans la conduite.

La célérité $a^{\prime}$ est du même ordre que dans le cas du réducteur sans diaphragmes.

En conclusion, on peut dire que l'expérience montre la nécessité de «cloisonner» le tuyau réducteur de célérité, même sur un troncon de conduite où la pression statique est quasi constante. 
2. - Essais de la série $I_{C}$ correspondant au deuxième régime (tuyau réducteur de célérité aplati par flambage de ses parois) :

Nous n'avons pu disposer de l'installation d'essai pendant un temps suffisant pour effectuer des expériences systématiques dans ce domaine. Les quelques enregistrements effectués montrent tous une réduction de célérité sensiblement plus grande que dans le premier régime et surtout une forte augmentation de l'amortissement.

La figure 19 reproduit, à titre d'exemple, les courbes de surpression aux sections d'abcisse $x=1,30 \mathrm{~m}$ et $x=72,30 \mathrm{~m}$ pour la « coupure» rapide d'un débit de $7,65 \mathrm{l} / \mathrm{s}$, la pression absolue de l'air à l'intérieur du réducteur de célérité étant de $14,85 \mathrm{~m}$ d'eau; avant le passage de la surpression, le tuyau de caoutchouc est done au voisinage du flambage; celui-ci survient au cours du coup de bélier.

On voit immédiatement que la surpression maximum est fortement réduite et que le coup de bélier est presque entièrement amorti au bout de la première demi-période. La célérité, calculée d'après la valeur du coup de bélier maximum par la formule $h-h_{0}=\frac{a \mathrm{~V}_{0}}{g}$ est de l'ordre de $100 \mathrm{~m} / \mathrm{s}$ seulement. L'efficacité du réducteur de célérité est bien mise en lumière par la comparaison des figures 15 et 19 ; si l'on remarque que le débit coupé est 6,6 fois plus grand dans ce dernier cas, on voit que le réducteur de célérité réduit ici au $1 / 13$ de sa valeur le coup de bélier maximum dans la conduite classique et assure son amortissement complet dans un temps très court.

Les enregistrements de la figure 19 ont été relevés avec un réducteur dépourvu de diaphragme; c'est ce qui explique les oscillations de grande fréquence que l'on remarque sur les diagrammes et la coexistence de deux ondes de surpression distinctes (dans l'air et dans l'eau), bien visible dans le deuxième diagramme de la figure 19.

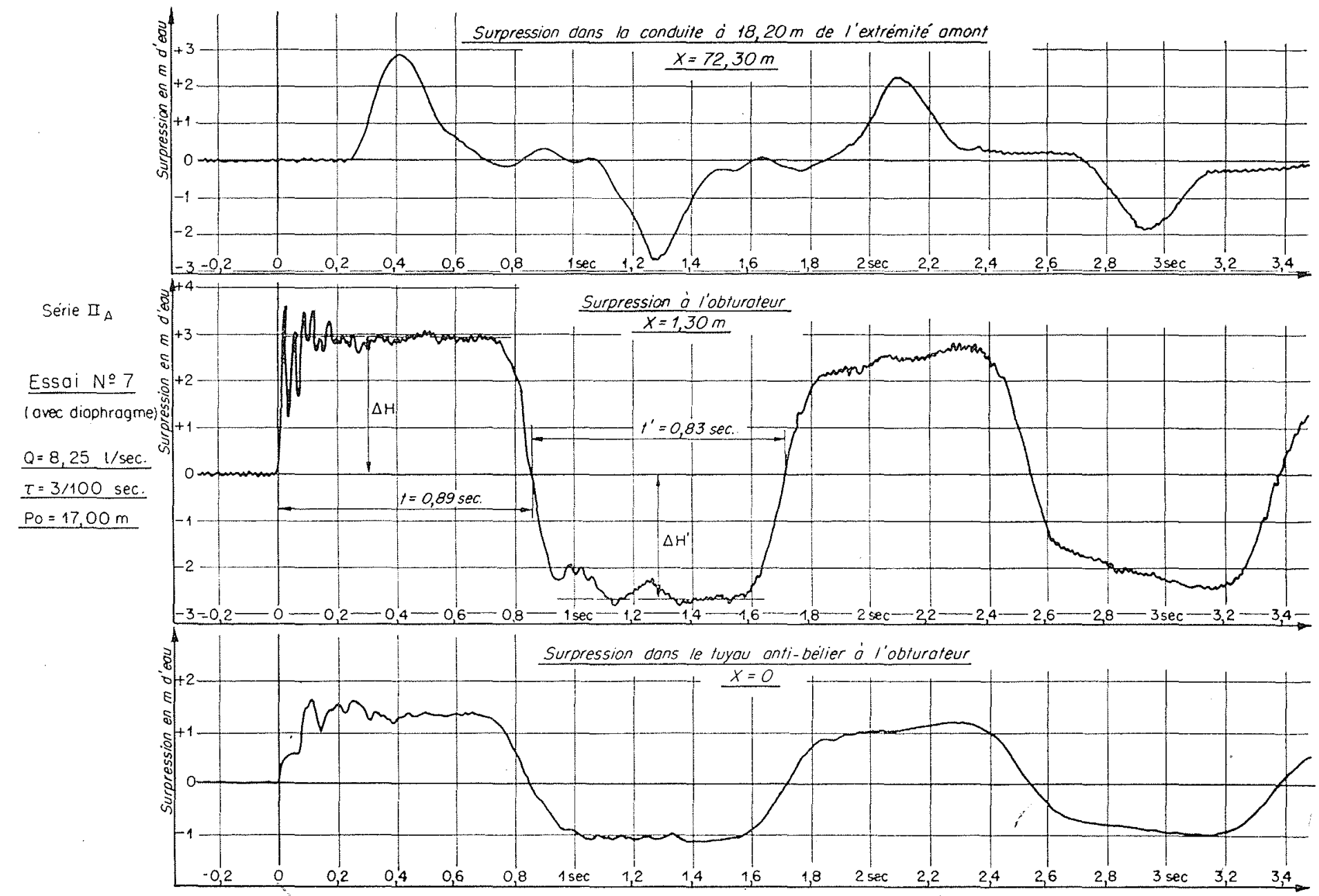

FIG. 18. 
Les expériences de la série $\mathrm{I}_{c}$ soulignent l'intérêt d'utiliser un tuyau réducteur de célérité fonctionnant au voisinage de sa limite de flambage; la section droite du tuyau pourra avantageusement être choisie de facon à favoriser ce flambage.

\section{3. - Essais de série $\mathrm{II}_{A \mathrm{C}}$ correspondant au régime de transition (tuyau réducteur de célérité tantôt en tension, tantôt en flambage au cours de la surpression):}

Ces essais ont donné des résultats assez dispersés mais les surpressions maxima constatées ont toujours correspondu i des célérités comprises entre celles de 100 et $170 \mathrm{~m} / \mathrm{s}$ trouvées pour: les deux régimes principaux étudiés ci-dessus.

\section{4. - Essais de "fermetures lentes":}

Nous n'avons pu disposer de l'installation d'essai pendant un temps assez long pour procéder à des essais systématiques sur les fermetures lentes.

Les quelques expériences effectuées confirment le résultat des calculs exposés dans le chapitre I; toutefois, en raison de l'amortissement ${ }^{1}$ très important produit par le réducteur de célérité, les coups de bélier «observés» sont plus faibles que ceux « calculés ».

La figure 20 reproduit l'enregistrement de la courbe de surpression à l'obturateur pour la coupure en 1,6 secondes d'un débit de $6,2 \mathrm{l} / \mathrm{s}$, la pression absolue de l'air dans le réducteur de

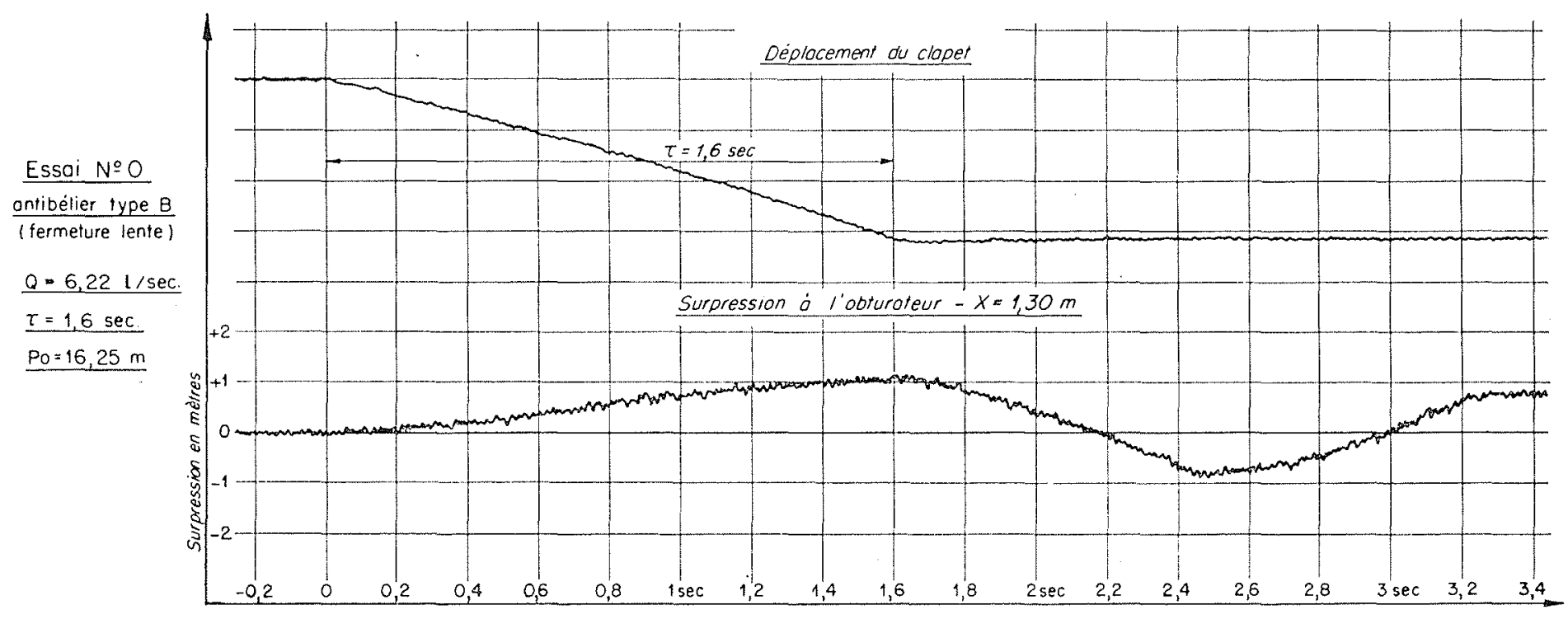

Frr. 20.

célérité (sans diaphragmes) étant de $16,25 \mathrm{~m}$ d'eau (premier régime).

\section{III. - Étude de la déformation du tuyau réducteur de célérité à compression d'air en régime statique.}

Par suite des contraintes mécaniques imposćes à la paroi de caoulchouc du réducteur, les formules établies au chapitre II, en supposant cette paroi infiniment souple el sans tension, ne s'appliquent pas rigoureusement aux conditions de nos expériences. Il est facile pourtant de les adapter à notre cas particulier en introduisant des paramètres représentant la déformation globale du réducteur de célérité en fonction de la pression; ces paramètres peuvent toujours, quelle que soit la complexité du système défor- mable, être mesurés au moyen d'un essai simple que nous allons décrire avec quelque détail en raison de son importance poụr l'éventuelle mise au point technologique d'un réducteur de célérité «industriel » de même type, mais de constitution quelconque.

a) Variation DE Yolume DU TUYaU DE CAOUTCHOUC REMPLI D'EAU EN FONCTION DE LA DIFFÉRENCE DE PRESSION $p=\mathrm{P}-\mathrm{H}$ :

Le tuyau de caoutchouc rempli d'eau est raccordé à un tube de verre calibré; le déplacement

1. Rappelons que la théorie d'Alzievi, négligeant toutes les pertes d'énergie dans l'eau et dans la paroi de la conduite, ne peut mettre en évidence un amortissement proprement dit. 
du ménisque dans ce tube de niveau permet moyennant des précautions convenables, de mesurer les variations de volume intérieur du tuyau lorsque l'on fait varier la pression de l'eau qu'il renferme (en faisant agir une pression d'air comprimé au-dessus du ménisque par exemple).

Si, pour une variation $\Delta p$ de la différence de pression $p$ entre l'intérieur et l'extérieur du tuyau, le volume de ce dernier passe de $\mathrm{V}$ à $V+\Delta V$, nous définirons son coefficient de déformation cubique par l'expression :

$$
\alpha=\frac{1}{\Delta p} \frac{\Delta V}{\mathrm{~V}}
$$

Pour des valeurs de $p==p-\mathrm{H}$ comprises entre 1 et $8 \mathrm{~m}$ d'eau, nous avons trouvé, à partir de la courbe expérimentale ${ }^{1}$ :

$$
\begin{gathered}
\frac{\Delta V}{V}=f(\mathrm{P}-\mathrm{H}): \\
\alpha=3,3 \% \text { par mètre d'eau }
\end{gathered}
$$

b) Varition dU volume du tuyau de caOdTCHOUC REMPLI D'UN CERTAIN VOLLME D'AIR $\left(V_{0}\right.$ A LA PRESSION $\left.P_{0}\right)$ LonsQUe LA presSION DE L'EAU QUI L'ENTOURE VARIE :

La variation de volume du réducteur de célérité sous l'effet d'une surpression dépend, non seulement de la déformation de sa paroi telle qu'elle est définie par l'expérience précédente donnant le coefficient $\alpha$, mais aussi de la compressibilité de l'air qu'il contient.

Supposons qu'à l'origine un tronçon du réducteur de célérité renferme un volume d'air $V_{0}$ à la pression absolue $\mathrm{P}_{0}$ et que le système soit en équilibre sous la pression absolue $\mathrm{H}_{0}{ }^{+}$de l'eau qui l'entoure. Le problème est de déterminer la variation de volume du réducteur de célérité lorsque la pression de l'eau prendra une valeur quelconque $\mathrm{H}^{+}$.

Expérimentalement, il est plus commode de déterminer tout d'abord la famille de courbes :

$$
\mathrm{P}=f\left(\mathrm{H}^{+}, \mathrm{P}_{0}\right)
$$

d'où l'on déduira, moyennant une hypothèse sur le mode d'évolution (isotherme ou adiabatique) de l'air, la famille de courbes :

$$
\mathrm{V}=f\left(\mathrm{H}^{+}, \mathrm{P}_{0}\right)
$$

1. En raison des grandes déformations du caoutchouc, a. ne peut être défini par :

$$
\alpha=\frac{1}{\Delta p} \frac{\Delta \mathrm{V}}{\mathrm{v}_{0}}
$$

$\mathrm{V}_{0}$ étant le volume initial correspondant par exemple à $\mathrm{P}-\mathrm{H}=\mathbf{0}$. qui permettra de déterminer les coefficients de déformation cubiques :

$$
\beta=\frac{1}{d \mathrm{H}} \frac{d \mathrm{~V}}{\mathrm{~V}}
$$

à introduire dans la formule donnant la célérité dans la conduite munie du tuyau anti-bélicr.

Si l'évolution de l'air est isotherme, un calcul simple montre que l'on peut admettre, en premiere approximation, dans les limites de nos essais :

$$
\beta=-\frac{\alpha}{1+\alpha P}
$$
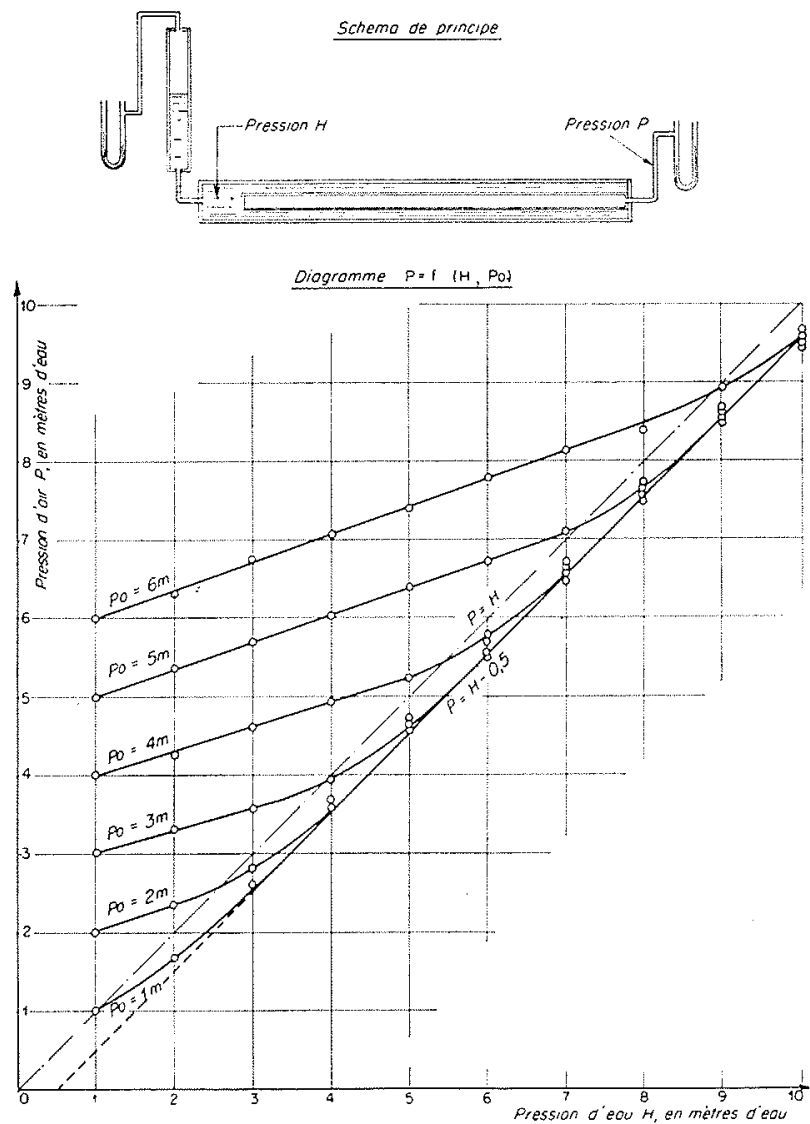

FIi. 21.

Essai de compressibilité statique du tuyau antibélier « $B$ ».

La figure 21 donne le schéma du dispositif expérimental utilisé pour le relevé des courbes $\mathrm{P}=f\left(\mathrm{H}^{+}, \mathrm{P}_{0}\right)$ et le diagramme obtenu (les pressions $\mathrm{P}_{0}$ et $\mathrm{H}$ sont, dans cette figure, comptées en mètres d'eau au-dessus de la pression atmosphérique).

Il apparaît immédiatement que la déformation du tuyau de caoutchouc se produit suivant deux régimes nettement différents suivant qu'il y a 
ou non flambage de sa paroi, ainsi que nous l'avons déjà indiqué au paragraphe II ci-dessus.

\section{1. - Premier régime :}

$$
\mathrm{H}<\mathrm{P}
$$

Lorsque la pression $\mathrm{P}$ de l'air à l'intérieur du tuyau est nettement plus grande que $H$ (points au-dessus de la droite $\mathrm{P}=\mathrm{H}$ ), les courbes $\mathrm{P}=f(\mathrm{H})$ pour des valeurs constantes de $\mathrm{P}_{0}$ sont des droites presque parallèles dans les limites de nos essais. Leur pente $\frac{d \mathbf{P}}{d \mathbf{H}}$ est de l'ordre de $1 / 3$, c'est-à-dire que l'accroissement de la pression de l'air dans le tuyau est environ trois fois moindre que celui de l'eau qui l'entoure.

Le tuyau restant de forme circulaire, puisque sa paroi est tendue, on peut calculer l'équation de la famille des courbes $\mathrm{H}^{+}=f\left(\mathrm{P}, \mathrm{H}_{0}\right)$; en admettant une évolution isotherme de l'air, on trouve :

$$
\mathrm{H}^{*}-\mathrm{H}_{0}{ }^{*}=\mathrm{P}-\mathrm{P}_{0}+\frac{2,3}{\alpha} \log \frac{\mathrm{P}}{\mathrm{P}_{0}}
$$

formule qui concorde bien avec les résultats expérimentaux que nous avons obtenus.

\section{2. - Deuxième régime :}

$$
\mathrm{H}^{+}+0,5>\mathrm{P}
$$

Lorsque la pression de l'eau est supérieure de plus de $0,5 \mathrm{~m}$ d'eau à la pression de l'air contenu dans le tuyau de caoutchouc, celui-ci s'aplatit par flambage de sa paroi; cette dernière n'intervient plus alors dans la relation $\mathrm{P}=f\left(\mathrm{H}^{+}\right)$ que pour imposer un écart constant de $0,5 \mathrm{~m}$ d'eau ${ }^{1}$ entre les deux pressions $\mathrm{P}$ et $\mathrm{H}^{+}$.

Quel que soit $P_{0}$, tous les points expérimentaux se placent sensiblement sur la droite :

$$
\mathbf{H}^{*}=\mathrm{P}+0,5
$$

\section{3. - Régime de transition :}

$$
\mathrm{P}<\mathrm{H}^{+}<\mathrm{P}+0,5
$$

La figure 21 montre que l'on passe progressivement du premier au deuxième régime décrit ci-dessus.

1. La pression de flambage de notre tube de caoutchouc calculée par la formule classique:

ressort à $0,46 \mathrm{~m}$ d'eau.
Considérons maintenant la relation

$$
\mathrm{V}=f\left(\mathrm{H}^{*}, \mathrm{P}_{0}\right)
$$

qui se déduit dans difficulté de la précédente. En effet, on a, en supposant par exemple la compression isotherme de l'air :

$$
V=\frac{1}{P} \cdot P_{0} V_{0}
$$

formule dans laquelle $P_{0}$ et $V_{0}$ sont connus et $P$ donné en fonction de $\mathrm{H}$ par le diagramme expérimental de la figure 21. Nous avons établi ainsi le diagramme $\mathrm{V}=f\left(\mathrm{H}^{+}, \mathrm{P}_{0}\right)$ donné par la fi-

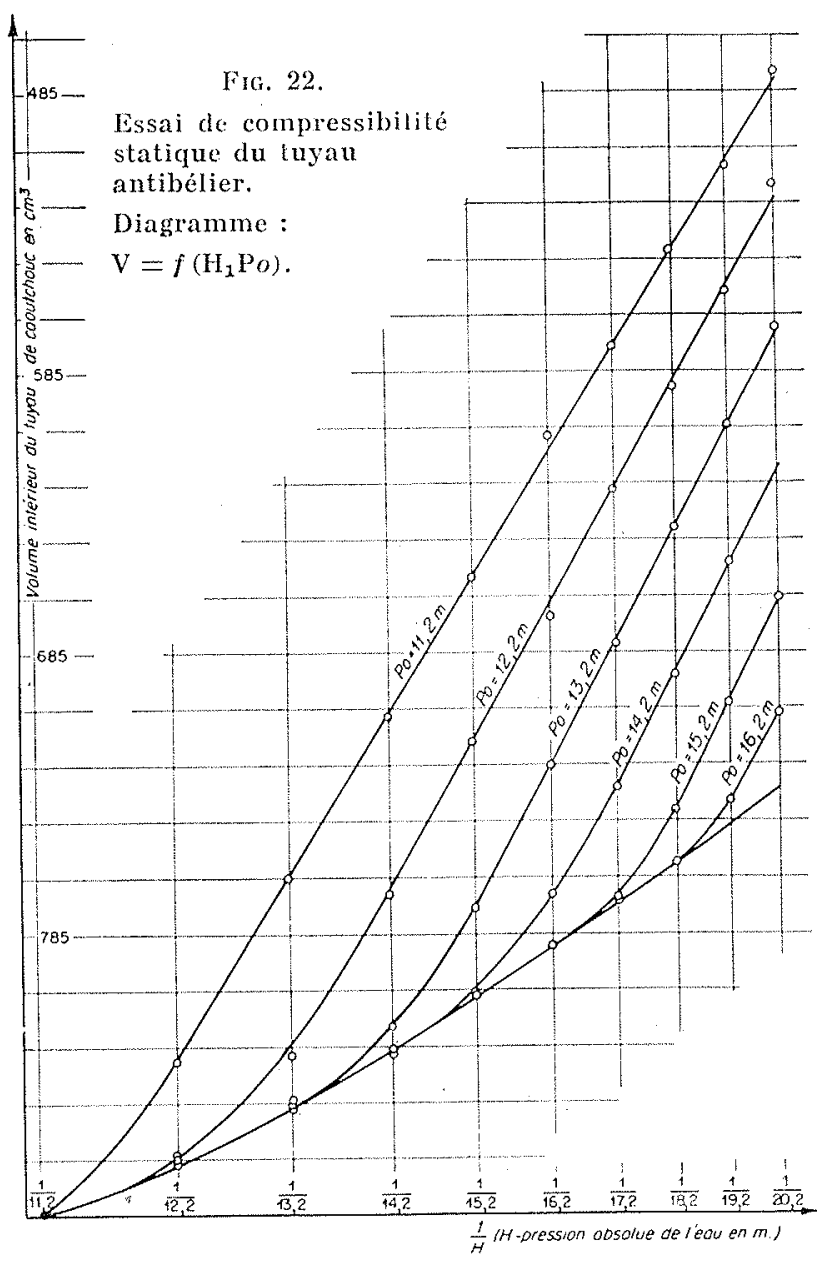

gure 22. Dans le deuxième régime. nous avons vu que $\mathrm{P}=\mathrm{H}^{+}-0,5$; done :

$$
V=\frac{1}{H-0,5} P_{0} V_{0}
$$

Pour plus de clarté, nous avons tracé le diagramme $V=f\left(\frac{1}{H}, P_{0}\right)$ pour $V_{0}$ constant. On voit que, dans le deuxième régime, l'expérience donne bien, comme le prévoit la formule ci-des- 
sus, une famille de droites cotées en $P_{0}$ Pour le premier régime, il se trouve que, quel que soit $\mathrm{P}_{0}$, dans la limite do nos essais, tous les points expérimentaux se groupent sur une courbe unique à laquelle viennent se raccorder, par une zone de transition, les droites afférentes au premier régime.

C'est sur le diagramme de la figure 22 que nous prendrons les éléments nécessaires au calcul dı coefficient de déformation cubique :

$$
\beta=\frac{1}{d \mathrm{H}} \frac{d \mathrm{~V}}{\mathrm{~V}}
$$

applicable dans chacune de nos expériences.

\section{IV. - Confrontation \\ des résultats "observés " et "calculés»}

Pour ne pas allonger démesurément notre exposé, nous nous bornerons àl'examen des essais relatifs aux « fermetures rapides » sur la conduite à réducteur de célérité à compression d'air.

Utilisant les notations de KorTewer, nous calculerons la célérité $a^{\prime}$ dans la conduite munie du réducteur de célérité à compression d'air par la formule :

$$
a^{\prime}=\frac{\left(\frac{g}{\varpi}\right)^{1 / 2}}{\left(\frac{1}{\varepsilon_{1}}+(1+c) \frac{1}{\varepsilon_{2}}+c \beta\right)^{1 / 2}}
$$

dans laquelle : $\frac{1}{\varepsilon_{1}}$ est le coefficient de compressibilité de l'eau que nous prendrons égal à $48,3 \times 10^{-10} \mathrm{~m}^{2} / \mathrm{kg}$.

$\frac{1}{\varepsilon_{2}}$ est la variation relative de volume de la conduite pour une variation de pression égale à l'unité; compte tenu de la flexion de la plaque portant le clapet obturateur aval $\frac{1}{\varepsilon_{2}}=18,4 \times 10^{10}$ $\mathrm{m}^{2} / \mathrm{kg}$ dans tous nos essais.

$\beta$ est la variation relative de volume du réducteur de célérité pour une variation de pression dans la conduite égale à l'unité; sa valeur sera déduite dans chaque cas du diagramme de la figure 22, ainsi qu'il a été expliqué plus haut :

$$
c=\frac{s}{s}=\frac{5,2}{452}=0,0115
$$

compte tenu de la déformation du tuyau de caoutchouc.

a) Essars DE LA SÉRIE $\mathrm{I}_{A}$ ET $\mathrm{II}_{\mathrm{A}}$ (en dehors de la zone de flambage du tuyau de caoutchouc):

Dans ces essais, la pression absolue initiale $\mathrm{P}_{0}$ de l'air a varié de 15,6 à $20,10 \mathrm{~m}$ d'eau; la valeur de $\beta$ correspondante est comprise d'après le diagramme de la figure 22 entre 2,2 et $2 \%$ par mètre d'eau, soit :

$$
\beta=2 \text { à } 2,2 \times 10^{-5} \mathrm{~m}^{2} / \mathrm{kg}
$$

La célérité théorique ressortirait donc à :

$$
\begin{aligned}
a^{\prime}= & \frac{9.900}{(48,3+18,6+2.530)^{1 / 2}} \\
= & 193 \mathrm{~m} / \mathrm{s} \text { pour } \beta=2,2
\end{aligned}
$$

et :

$$
203 \mathrm{~m} / \mathrm{s} \text { pour } \beta=2,0
$$

Or, la moyenne des célérités relevées au cours de cette série d'essais s'établit à :

$$
\begin{aligned}
& a_{h^{\prime}}=170 \mathrm{~m} / \mathrm{s} \\
& \quad\left(\text { calcul par la formule } h-h_{0}=\frac{a \mathrm{~V}_{0}}{g}\right)
\end{aligned}
$$

et :

$a_{\mathrm{T}^{\prime}}=210 \mathrm{~m} / \mathrm{s}$
$\quad\left(\right.$ calcul d'après la période $\frac{4 \mathrm{~L}}{a}$ mesurée $)$

Les célérités calculées et observées sont bien du même ordre de grandeur; il semble normal que $a_{h}$ soit inférieur à la célérité théorique, en raison des phénomènes d'amortissement qui ne sont pas pris en compte par cette dernière.

\section{b) ESSAIS DE LA SÉRIE $\mathrm{II}_{\mathrm{C}}$ (tuyau de caoutchouc aplati par flambage) :}

Dans ces essais, la pression absolue de l'air est voisine de $14,9 \mathrm{~m}$ d'eau. La paroi du tuyau intervient donc peu et on a sensiblement :

$$
\beta=\frac{1}{\mathrm{P}} \# \frac{1}{\mathrm{P}_{0}}
$$

car si la compression est isotherme :

$$
\frac{d \mathrm{~V}}{\mathrm{~V}}=\frac{d \mathrm{P}}{\mathrm{P}}
$$

d'où :

$$
\beta=\frac{1}{d \mathrm{H}} \frac{d \mathrm{~V}}{\mathrm{~V}}=\frac{1}{d \mathrm{H}} \frac{d \mathrm{P}}{\mathrm{P}} \# \frac{1}{\mathrm{P}}
$$

C'est bien ce que confirme le diagramme de la figure 22 ; nous prendrons donc :

$$
\beta=\frac{1}{14,9}=6,7 \% \text { pár mètre d'eau }
$$

ou :

$$
\beta=6,7 \times 10^{-5} \mathrm{~m}^{2} / \mathrm{kg}
$$


ce qui donne comme valeur théorique de la célérité $a^{\prime}$ :

$$
a^{\prime}=\frac{9.900}{(48,3+18,6+6.780)^{1 / 2}}=120 \mathrm{~m} / \mathrm{s}
$$

Or, l'expérience a conduit aux chiffres suivants :

$$
a_{h}^{\prime}=93 \mathrm{~m} / \mathrm{s} \quad \text { et } \quad a_{\mathrm{TI}}^{\prime}=\mathbf{1 0 3 \mathrm { m } / \mathrm { s }}
$$

L'amortissement considérable que montro l'enregistrement de la figure 19 explique proba- blement l'écart entre les célérités observées et calculées.

En conclusion, on peut dire que le schéma, quelque peu grossier, admis pour établir les formules du chapitre II, donne une approximation acceptable - et dans le sens de la sécurité - pour le calcul de la célérité d'une conduite munie d'un réducteur de célérité. La détermination expérimentale du coefficient $\beta$ suivant le procédé exposé page 191 permet ce calcul quelle que soit la complexité de la loi reliant la variation de volume du réducteur à la surpression.

\section{CONCLUSION}

Dans le cas simple d'une conduite à « caractéristique unique» soumise à des «manœuvres linéaires », nous avons montré, à l'aide de la théorie d'Alliḱvi, que la réduction de la célérité des ondes élastiques entraîne toujours une diminution, dans les mêmes proportions, des coups de bélier de fermeture rapide. Pour les fermetures lentes, la réduction de la célérité n'est susceptible de permettre une économie sur le dimensionnement de la conduite que dans

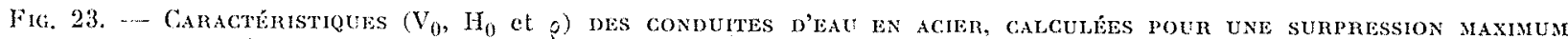

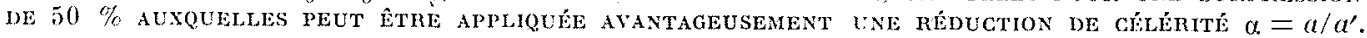

\section{Fermetures Lentes}

$1^{\circ}$ Une réduction de célérité $\alpha_{i}$ entraine une diminution du coup de bélier « maximum possible $\gg$ pour toutes les conduites dont le point de fonctionnement a pleine charge se trouve à gauche de l'ordonnée —_- coté $\alpha_{i}$, si :

$$
\theta_{0}<\alpha_{i}
$$

$2^{\circ}$ Pour des conduites calculées pour une surpression égale a $50 / n \%$ de $\mathrm{H}_{0}$, on pourra utiliser la même abaque en divisant par $n$ les cotes $\alpha_{i}$ ci-dessus.

N. B. - L'épaisseur de la conduite correspond a un taux de travail de la tôle de $10 \mathrm{~kg} / \mathrm{mm}^{2}$ sous la pression $\mathrm{H}_{0}$.

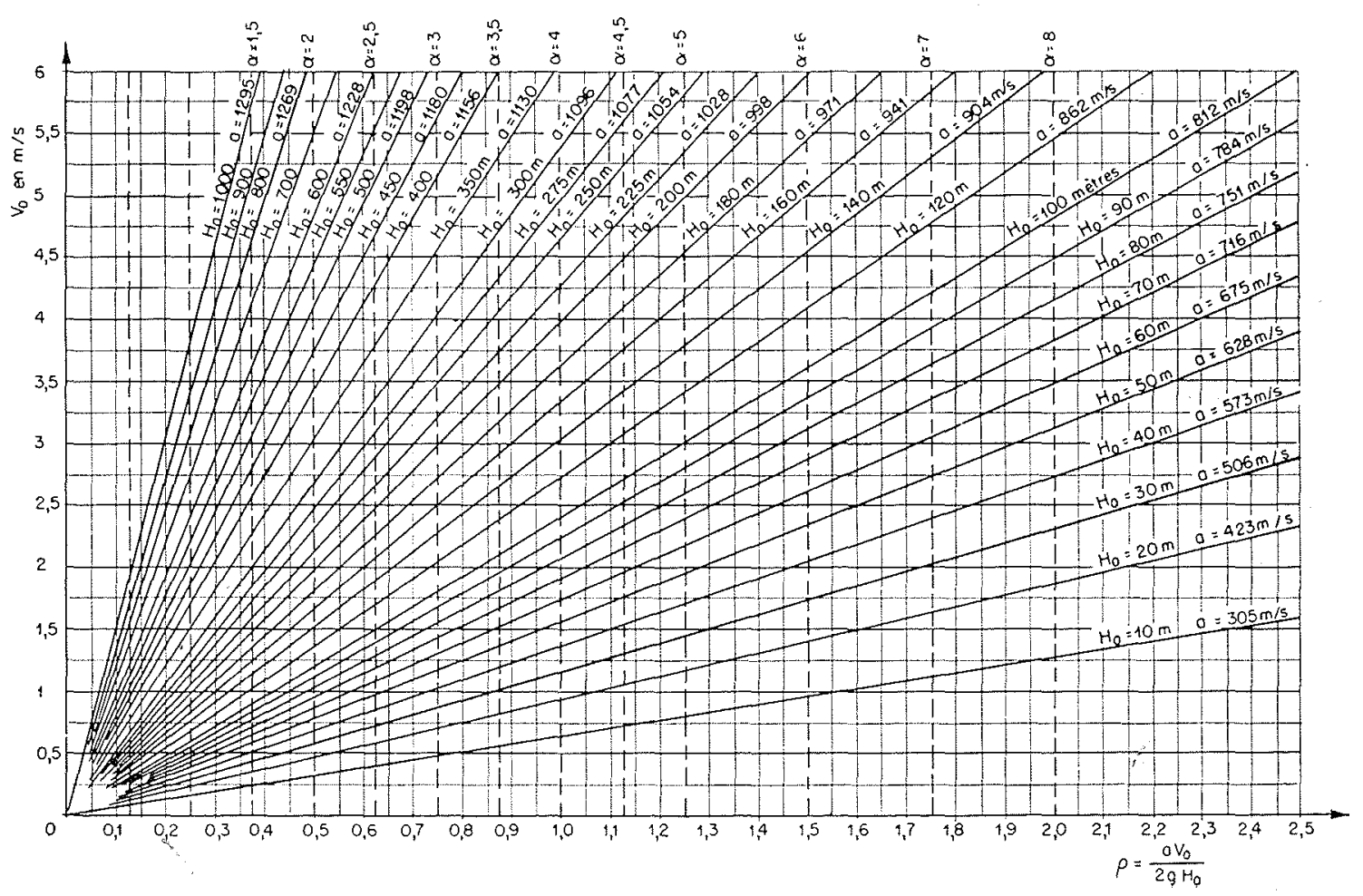


certains cas particuliers que nous avons précisés. Les cas plus complexes de conduites à caractéristiques variables, de réseaux de conduites, de fermetures non linéaires, etc..., devront être étudiés individuellement par la méthode graphique de M. BErgenon.

Les essais effectués au Laboratoire national d'Hydraulique de Chatou ont montré l'efficacité de l'un des types de réducteur de célérité que nous avons proposé et le degré d'approximation - suffisant en pratique - des formules que nous avons établies pour son calcul. La réduction observée sur les coups de bélier est toujours supérieure à celle donnée par les formules théoriques, sans doute parce que celles-ci ne tiennent pas compte de l'amortissement considérable des surpressions dî aux pertes d'énergie consécutive à l'action du réducteur de célérité.

Quel est le champ d'application pratique éventuel des réducteurs de célérité? Les «manouvres rapides» pour lesquelles ils constituent des anti-béliers très efficaces sont rarement utilisćes ${ }^{1}$ dans les appareils industriels; le «déclenchement» du moteur électrique de commande d'une pompe refoulant sur une longue conduite constitue pourtant un exemple fréquent de ce genre de manœuvre; il serait intéressant d'étudier dans ce cas la rentabilité du nouveau dispositif de protection concurremment avec les anciens : volants, réservoirs d'air, etc...

1. Elles peuvent pourtant se produire inopinément par aceident ou fausse mancuvre.
Pour les «manœuvres lentes» telles que celles auxquelles sont soumises les conduites forcées d'usines hydro-électriques, le champ d'application paraît assez étroit; il se situe dans le domaine des chutes moyennes où il sera parfois possible (pour une même durée de manœuvre en secondes) de transformer une «fermeture lente» en «fermeture rapide» plus avantageuse, grâce au réducteur de célérité. (Voir l'abaque de la figure 23.)

Dans le cas de conduites bifurquées ou de réseaux de conduite, la possibilité de faire varier la célérité propre à telle branche de canalisation pourra permettre à l'Ingénieur, bien familiarisé avec la méthode graphique de M. Bergrnon, de diminuer les surpressions en certains points criticues.

Le réducteur de célérité pourra encore être utilisé pour atténuer ou supprimer les surpressions —. et les vibrations corrélatives —ayant pour origine des phénomènes de résonance entre la période propre de tout ou partie d'une conduite et celle des organes qui lui sont reliés (régulateurs, pompes, organes émetteurs de tourbillons périodiques, etc...). La simplicité de mise en place du système, même dans une installation existante, sera alors un grand avantage.

Enfin, les laboratoires pourraient employer le réducteur de célérité pour l'étude, sur modèle réduit, des coups de bélier dans des systèmes complexes.

\section{I S CUSSION}

M. Ie Président Paul Bergeron remercie M. RÉménúras de son étude qui met en lumière :

1. L'influence d'une réduction de la célérité sur le coup de bélier dans le cas d'une conduite à caractéristique unique et des fermetures linéaires;

$2^{\circ}$ Les cas dans lesquels une telle réduction conduit à une diminution du coup de bélier;

$3^{\circ}$ Un dispositif original permettant de réduire la célérité.

M. Fenrañ signale que l'emploi des aciers à haute résistance dans la construction des conduites forcées a conduit à une certaine réduction de la célérité. En effet. le taux de travail admis dans certaines conduites autofrettées ou surpressées dépasse $30 \mathrm{~kg} / \mathrm{mm}^{2}$ au lieu de de $8 \mathrm{~kg} / \mathrm{mm}^{2}$ dans les anciennes conduites soudées en acier doux. La déformation correspondant à une mème surpression est donc, à égalité de module élastique environ 4 fois plus forte dans les conduites modernes et c'est ee qui explique les réductions de célérité constatées.

M. Danel remarque que l'étude de M. RÉméntétas est basce sur la thérie d'Alliévi qui s'applique au cas de la fermeture d'un orifice suivant une loi lineaire. Pour les fermetures lentes $(\theta>1)$ on peut montrer qu'en s'écartant assez peu d'une loi de fermeture linéaire on obtient des valeurs maxima du coup de bélier assez différentes de celles d'Alliévi; dans de tels cas une augimentation do célérité peut conduire à une diminution du coup de bélier.

Par contre la réduction de la célérité réduit toujours les coups de bélier de fermeture rapide $(\Theta<1)$ mais dans les apparcils industriels on utilise surtout des fermetures lentes. Pour tirer lá quintessence du procéde proposé par M. RËmÉxiéras, il est indispensable d'étudier chaque installation par des methodes tenant compte de linfluence des caractéristiques de la fermeture sur la valeur maximum du coup de bélier.

M. RËméneras répond qu'il a basé son exposé sur la théorie d'Alliévi pour les conduites à caractéristique unique et à fermeture linéaire parce qu'elle est la seule qui permette un exposé d'ensemble assez simple de l'influence d'une réduction de la célérité sur les phénomènes de coup de bélier. Il a signalé d'aillenrs que les cas plus complexes qui sortent du cadre de cette théorie pouvaient être traités par la méthode graphique de M. Bengeron; on pourra ainsi se rendre compte qu'il n'est pas toujours nécessaire de disposer le réducteur de célérité dans tous les troncons d'un système complexe de conduites; il est bien d'accord avec M. Daner sur le fait que cest senlement dans le cas d'une fermeture rapide que le coup de bélier est à coup sûr diminué par une réduction de célérité; ce type de manceuve est assurément pou fréquent dans les appareils industriels; il 
est souvent réalisé pourtant - saut dispositif spécial - an moment du déclenchement du moteur électrique commandant une pompe centrifuge. On peut aussi penser que certaines variations faibles mais rapides de régime engendrant des vibrations gênantes sinon dangereuses pourraient ètre amorties par réduction de célérité. Pour les fermetures lentes, il y a lieu d'étudier chaque cas particulier; les résultats obtenus dans le cadre simplifié de la théorie d'Alliévi montrent que suivant le cas il peut y avoir augmentation ou diminution du coup de béliex maximum: le plus souvent le coup de bélier restera inchangé. Même dans les cas théoriquement favorables il sera, bien entendu, nécessaire d'étudier l'intérêt économique d'une réduction de la célérité par rapport à d'autres dispositifs, de protection contre les coups de bélier.

M. Darrieus signale que l'effet du dispositif proposé par M. Réménitras en vue de réduire les coups de bélier, dépend en fait de l'impédance d'onde et non de la vitesso de propagation; la réduction de la vitesse n'est d'ailleurs pas avantageuse en principe, car elle prolonge la durée de l'adaptation en différant la répercussion naturellement favorable qu'exercent sur l'ensemble du phénomène, pour les fermetures pas trop rapides, les conditions à l'entrée de la conduite. D'autre part, M. DARRueus craint que ce dispositif nécessairement un peu fragile ne comporte une autre sujétion dans la difficulté des calculs due au caractère non lineaire de son fonctionnement et à la disproportion entre les diametres $d$ et D. Par exemple il ressort d'un calcul simple que, pour une surpression déterminant dans la paroi une extension de 1/1.000 dans l'acier (20 mapz), soit une augmentation de volume ou de section de 2 pour 1.000 , le diamètre du petit tuyau devrait atteindre $1 / 20$ du grand pour que son volume équivaille à cette seule augmentation élastique de volume. De plus, l'augmentation élastique est linéaire, tandis que la contraction du tube à air comprimé est soumise à une loi plus compliquée.
M. Rémḱniéras répond qu'il a donné des abaques permettant de se rendre compte de la réduction de célérité obtenue en fonction du rapport $c$ des sections du tuyau réducteur de célérité et de la conduite, d'une part, et de la pression de service, d'autre part; pour $c=5 \%$ la célérité est réduite au $1 / 6$ environ de sa valeur initiale pour une pression de service de $100 \mathrm{~m}$ d'eau et au $1 / 4$ pour une pression de $500 \mathrm{~m}$ d'eau 1 . Les expériences faites au Laboratoire de Chatou avec $c \neq 1 \%$ donnent des réductions de célérité de l'ordre de $1 / 10$ pour une pression de $5 \mathrm{~m}$ d'eau. En ce qui concerne la difficulté des calculs, M. Réméñéras observe que, quelle que soit la complexité du système réducteur de célérité, il est toujours possible d'établir expérimentalement la courbe donnant sa variation de volume en fonction de la pression, ainsi que cela a été fait dans les expériences ci-dessus,

M. le Président pense que le dispositif réducteur de célérité est indiscutablement intéressant du point de vue théorique pour diminuer les coups de bélier engendrés par une variation de résime survenant en un temps inférieur à $2 \mathrm{~L} / a$. De telles variations sont évidemment beaucoup plus rare pour les turbines que pour les pompes qui donnent en cas de « déclenchement 》 une dépression de «fermeture rapide » dans la conduite de refoulement. Les expériences de M. RÉMÉNiÉRAs montrent qu'un tuyau « réducteur de célérité » de faible diamètre permet de réduire la célérité dans une proportion voisine du $1 / 5$; il sera intéressant de se rendre compte, dans chaque cas de la pratique, si ce dispositif est économiquement plus avantageux que ceux utilisés normalement : réservoirs d'air, volant, cheminée d'équilibre, etc...

1. Ces chiffres s'entendent pour un tuyau rempli d'air comprimé; la substitution à l'air d'un gaz liquéfiable serait sans doute tres avantageuse.

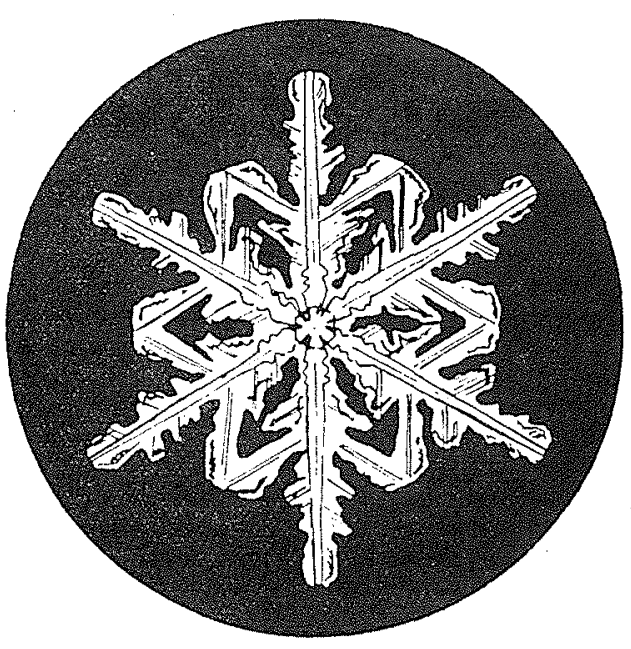

